\title{
Functions of a Glucan Synthase Gene GFGLS in Mycelial Growth and Polysaccharide Production of Grifola frondosa
}

Feng-Jie Cui a, c,*, Xi-Hong Wu a , Ting-Lei Tao a, Xin-Yi Zan a,*, Wen-Jing Sun a, c*, Da-Shuai $\mathrm{Mu}^{\mathrm{d}}$, Yan Yang ${ }^{\mathrm{b}}$, Di Wu

a. School of Food and Biological Engineering, Jiangsu University, Zhenjiang 212013, P.R. China

b. National Engineering Research Center of Edible Fungi, Shanghai Academy of Agricultural Sciences, Shanghai 201403, PR China

c.Jiangxi Provincial Engineering and Technology Center for Food Additives Bio-production, Dexing 334221, P.R. China

d. College of Marine Science, Shandong University (Weihai), Weihai 264209, PR China

* Corresponding Author: Phone: +86-511-88780226; Fax: +86-511-88780201; E-mail: fengjiecui@163.com; sunwenjing1919@163.com; zxy19880920@163.com. 


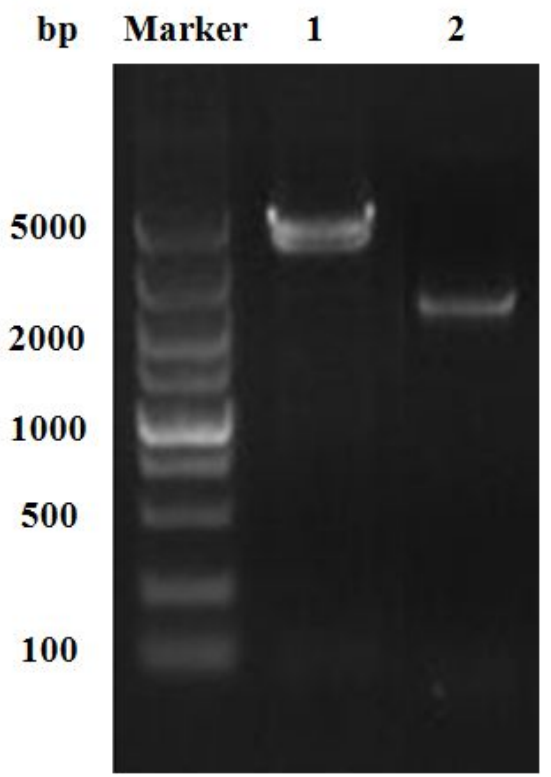

Figure S1 PCR amplification of two gene fragments (GFGLS1 and GFGLS2) in G. frondosa GF9801. The number of 1 and 2 represents GFGLS1 and GFGLS2, respectively. 
1 TGCGCGATTAGAGGCATTAGAGGTGAAGTTGCGAAACAGTAAAGCCAACTCGAGGAGAAA 61

GCAATACAGATAGAATATAGACAGACTAGATGCACGAATGAAACAAAAGACAAAAGGGCG

121

GGAATATGGCGTTGGCCAAGCATTAGCCATAGCCGCGATAAGTATGCGGCCCCAGACCTC 181

CGAAATTTCCTTATGTGGCCTGGTCGGTGGACTAACCAGTCTGAGACACCCTGGTTGCTG

241

CGTGCCGTGAGGCTGGGAGCAGGTTTGAACAGCGTCACTCCACTCTTTTCTCTTCATCGT

301

TCTCGCTGGTATTCTCCACACCGCTCACGGCGACGACCTTGTCACCTCTCTAGCGCACAT 361

GGCCGGTCGCTCTGGTCCACGAAGCCAGGAGGGCATGTATGTCTCCTCGTCTCCGTCTTC

$\begin{array}{lllllllllllllllllllll}1 & M & A & G & R & S & G & P & R & S & Q & E & G & M & Y & V & S & S & S & P & S\end{array}$ $\mathrm{S}$

421

AGACCCCCACGATCCCTTCAGCAACGTCCACGCACAAATGCCCGAGCCCCAGAGGTACTA

$\begin{array}{lllllllllllllllllllll}22 & \mathrm{D} & \mathrm{P} & \mathrm{H} & \mathrm{D} & \mathrm{P} & \mathrm{F} & \mathrm{S} & \mathrm{N} & \mathrm{V} & \mathrm{H} & \mathrm{A} & \mathrm{Q} & \mathrm{M} & \mathrm{P} & \mathrm{E} & \mathrm{P} & \mathrm{Q} & \mathrm{R} & \mathrm{Y}\end{array}$ $\mathrm{Y}$

481

CGACAACGACTCTGACCAGATAGATAATTACGGTCGGCGCGACACCTATGGCTCGGATGG

$\begin{array}{lllllllllllllllllllll}42 & \mathrm{D} & \mathrm{N} & \mathrm{D} & \mathrm{S} & \mathrm{D} & \mathrm{Q} & \mathrm{I} & \mathrm{D} & \mathrm{N} & \mathrm{Y} & \mathrm{G} & \mathrm{R} & \mathrm{R} & \mathrm{D} & \mathrm{T} & \mathrm{Y} & \mathrm{G} & \mathrm{S} & \mathrm{D}\end{array}$ G

541

CAGTAATGGAGGAAACGACGACGATCGTTATTATGACCACAACGGCGCCTACGATCCGTA

$\begin{array}{lllllllllllllllllllll}62 & \mathrm{~S} & \mathrm{~N} & \mathrm{G} & \mathrm{G} & \mathrm{N} & \mathrm{D} & \mathrm{D} & \mathrm{D} & \mathrm{R} & \mathrm{Y} & \mathrm{Y} & \mathrm{D} & \mathrm{H} & \mathrm{N} & \mathrm{G} & \mathrm{A} & \mathrm{Y} & \mathrm{D} & \mathrm{P}\end{array}$ Y

601

TGGCCGTGAGTGCCTTTTCCTCATGTTTCCATTCTCTGTGAGCTTAGTTTCCCCCAGCAC

$82 \quad \mathrm{G}$

661

AACCCGACACCGACTCGGACGTCGATGTTTACGGTCAGAAGTATGCCCCCTCAGCGGAGT

$\begin{array}{lllllllllllllllllllllll}84 & \mathrm{Q} & \mathrm{P} & \mathrm{D} & \mathrm{T} & \mathrm{D} & \mathrm{S} & \mathrm{D} & \mathrm{V} & \mathrm{D} & \mathrm{V} & \mathrm{Y} & \mathrm{G} & \mathrm{Q} & \mathrm{K} & \mathrm{Y} & \mathrm{A} & \mathrm{P} & \mathrm{S} & \mathrm{A} & \mathrm{E}\end{array}$ 721

CTCTTGGCCCTCCTCGCGTGGGCGTCTCAGAGTCCTCCACCCCGACTTTCATTGATCACA

$\begin{array}{llllllllllllllllllllll}104 & S & L & G & P & P & R & V & G & V & S & E & S & S & T & P & T & F & I & D & H\end{array}$ 781

ATGGTTCCGGCGGGCGAGAGCCATACCCAGCTTGGAGTTCCGAGCGCCAAATTCCACTAT

$\begin{array}{llllllllllllllllllllll}124 & N & G & S & G & G & R & E & P & Y & P & A & W & S & S & E & R & Q & I & P & L\end{array}$ 841

CGAAGGAGGAGATCGAGGACATCTTCCTGGACCTTACGCAGAAGTTTGGTTTTCAGCGGG

$\begin{array}{llllllllllllllllllllllll}144 & S & K & E & E & I & E & D & I & F & L & D & L & T & Q & K & F & G & F & Q & R\end{array}$ 901

ATTCCATGCGGAACATGGTATGCTTCTGGGTGAAAGCTATCTGGGTTGAATTCTTACACA 


\section{1}

AGTCTTTAGTTCGACTTTACGATGCAGCTACTGGATAGTCGCGCCTCGCGCATGTCGCCT

170

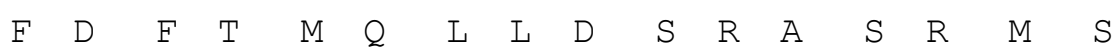

$\mathrm{P}$

\section{1}

AATCAGGCTCTCCTCACTTTGCATGCTGATTACATCGGTGGCCAGCACGCAAATTACCGC

$\begin{array}{lllllllllllllllllllll}187 & \mathrm{~N} & \mathrm{Q} & \mathrm{A} & \mathrm{L} & \mathrm{L} & \mathrm{T} & \mathrm{L} & \mathrm{H} & \mathrm{A} & \mathrm{D} & \mathrm{Y} & \mathrm{I} & \mathrm{G} & \mathrm{G} & \mathrm{Q} & \mathrm{H} & \mathrm{A} & \mathrm{N} & \mathrm{Y}\end{array}$ $\mathrm{R}$

1061

AAGTGGTACTTCGCTGCCCAGCTGGATCTCGACGACGCTATTGGACAGACACAAAACCCC

$\begin{array}{llllllllllllllllllllll}207 & \text { K } & \text { W } & \text { Y } & \text { F } & \text { A } & \text { A } & \text { Q } & \text { L } & \text { D } & \text { L } & \text { D } & \text { D } & \text { A } & \text { I } & G & Q & \text { T } & Q & \text { N }\end{array}$ $\mathrm{P}$

1121

GGTTTAAGGTCTACAAAGCGCAAGGGACACAAGGCCGTGGAAAAGTCCCTGAACATCGCT $\begin{array}{llllllllllllllllllll}227 & G & L & R & S & T & K & R & K & G & H & K & A & V & E & K & S & L & N & I\end{array}$ A

1181

CTGGACCGATGGCGGCAGGCCATGAACAACATGAGCCAGTACGACAGGATGCGACAGATC $\begin{array}{lllllllllllllllllllllll}247 & \text { L } & D & R & W & R & Q & A & M & N & N & M & S & Q & Y & D & R & M & R & Q\end{array}$ I

1241

GCACTATATCTCCTTTGCTGGGGCGAAGCTGCGCAGGTGCGATTTGTCCCAGAATGCCTA $\begin{array}{lllllllllllllllllllllllllll}267 & A & L & Y & L & L & C & W & G & E & A & A & Q & V & R & F & V & P & E & C\end{array}$ L

1301

TGTTTCATCTTCAAGTGTGCAGACGACTACTACCGATCGCCGGAGTGCCAGAGTCGGATA

$\begin{array}{llllllllllllllllllll}287 & C & F & I & F & K & C & A & D & D & Y & Y & R & S & P & E & C & Q & S & R\end{array}$ I

1361

GATCCCGTGCCAGAAGGCCTGTACTTGCATGCCGTCATCAAGCCCCTGTACAGGTTTATT $\begin{array}{lllllllllllllllllllll}307 & \text { D } & \text { P } & \text { V } & \text { P } & \text { E } & \text { G } & \text { L } & \text { Y } & \text { L } & \text { H } & \text { A } & \text { V } & \text { I } & \text { K } & \text { P } & \text { L } & \text { Y } & \text { R } & \text { F }\end{array}$ I

1421

CGCGACCAAGGTTACGAGGTAGTCGATGGGAAGTTTGTCCGACGGGAGAAGGACCATGAC

$\begin{array}{llllllllllllllllllll}327 & R & D & Q & G & Y & E & V & V & D & G & K & F & V & R & R & E & K & D & H\end{array}$ $\mathrm{D}$

1481

ACAATTATCGGGTACGACGATGTGAACCAGCTATTTTGGTATCCGGAGGGCATCGCTCGA $\begin{array}{llllllllllllllllllll}347 & \text { T } & \text { I } & \text { I } & G & Y & D & D & \text { V } & \text { N } & \text { Q } & \text { L } & \text { F } & \text { W } & \text { Y } & \text { P } & \text { E } & \text { G } & \text { I } & \text { A }\end{array}$ $\mathrm{R}$

1541

ATTGTCCTCGCGGACAAGGTATGCCGTCTGACATATACTTTTCTGGTGTTACTCATGCCA

367 I $\quad V \quad$ L $\quad$ A 
1601

TCTACGTATACAGACACGACTGGTCGACTTGGCACCAGCGCAACGTTTCATGAAATTTGA

$\begin{array}{lllllllllllllllll}373 & \text { T } & \text { R } & \text { L } & \text { V } & \text { D } & \text { L } & \text { A } & \text { P } & \text { A } & \text { Q } & \text { R } & \text { F } & \text { M } & \text { K } & \text { F }\end{array}$ D

\section{1}

CCGGATCGACTGGAACCGTGCATTCTTTAAGACGTACTATGAGAAACGATCTTTCGGCCA

$\begin{array}{llllllllllllllllllll}389 & R & I & D & W & N & R & A & F & F & K & T & Y & Y & E & K & R & S & F & G\end{array}$ $\mathrm{H}$

1701

CTTGCTTGTGAACTTTAACAGGATATGGGTCATTCATGTGTCCATGTACTGGTACTACAC

$\begin{array}{llllllllllllllllllll}409 & \mathrm{~L} & \mathrm{~L} & \mathrm{~V} & \mathrm{~N} & \mathrm{~F} & \mathrm{~N} & \mathrm{R} & \mathrm{I} & \mathrm{W} & \mathrm{V} & \mathrm{I} & \mathrm{H} & \mathrm{V} & \mathrm{S} & \mathrm{M} & \mathrm{Y} & \mathrm{W} & \mathrm{Y} & \mathrm{Y}\end{array}$ $\mathrm{T}$

1761

GGCTTACAACTCACCAACCGTCTACAATGGCATCAGGTCAACAGCGATGCGATGGTCGGC

$\begin{array}{lllllllllllllllllllll}429 & A & Y & N & S & P & \text { T } & \text { V } & \text { Y } & \text { N } & \text { G } & \text { I } & \text { R } & \text { S } & \text { T } & \text { A } & \text { M } & \text { R } & \text { W } & \text { S }\end{array}$ A

1821

AACTGCGCTCGGAGGCGCAGTTGCCACTGTGATCATGATCTTGGCGACGCTCGCCGAATT

$\begin{array}{llllllllllllllllllll}449 & \text { T } & \text { A } & \text { L } & G & G & A & \text { V } & \text { A } & \text { T } & \text { V } & \text { I } & \text { M } & \text { I } & \text { L } & \text { A } & \text { T } & \text { L } & \text { A } & \text { E }\end{array}$
$\mathrm{F}$

\section{1}

TTCGTACATTCCGACAACATGGAACAATACCTCGCATCTCACTCGACGTCTCATTTTCCT

$\begin{array}{llllllllllllllllllll}469 & \mathrm{~S} & \mathrm{Y} & \mathrm{I} & \mathrm{P} & \mathrm{T} & \mathrm{T} & \mathrm{W} & \mathrm{N} & \mathrm{N} & \mathrm{T} & \mathrm{S} & \mathrm{H} & \mathrm{L} & \mathrm{T} & \mathrm{R} & \mathrm{R} & \mathrm{L} & \mathrm{I} & \mathrm{F}\end{array}$ L

1941

CGCCATTACCCTTGCCCTCACGGCCGGTCCCACGTTTTACATCGCGATCGCCGAGAGCAG

$\begin{array}{llllllllllllllllllll}489 & A & I & T & L & A & L & T & A & G & P & T & F & Y & I & A & I & A & E & S\end{array}$
$\mathrm{S}$

\section{1}

CTCTCCCGGCGGCTCGTTGGCCCTCATTCTCGGCATTGTCCAATTTTTCATCTCGGTCGT

$\begin{array}{llllllllllllllllllllllll}509 & S & P & G & G & S & L & A & \text { L } & \text { I } & \text { L } & G & \text { I } & \text { V } & \text { Q } & \text { F } & \text { F } & \text { I } & S & \text { V }\end{array}$
$\mathrm{V}$

2061

CGCGACGCTGCTGTTCGCGTTCCTGCCCTCTGGACGCATGTTCGGCGATAGGGTGGCCGG

$\begin{array}{llllllllllllllllllll}529 & \text { A } & \text { T } & \text { L } & \text { L } & \text { F } & \text { A } & \text { F } & \text { L } & \text { P } & \text { S } & G & \text { R } & \text { M } & \text { F } & G & \text { D } & \text { R } & \text { V } & \text { A }\end{array}$ G

\section{1}

CAAGTCTCGGAAGTATCTGGCAAGTCAGACGTTCACGGCCAGCTACCCGACGCTGAAGGC

$\begin{array}{lllllllllllllllllllll}549 & K & S & R & K & Y & \text { L } & \text { A } & \text { S } & \text { Q } & \text { T } & \text { F } & \text { T } & \text { A } & \text { S } & \text { Y } & \text { P } & \text { T } & \text { L } & \text { K }\end{array}$ A

2181

ATCAGCACGGCTGGCTTCCGTGTGCCTCTGGATTCTCGTCTTCGGCTGCAAGTTCACCGA

$\begin{array}{llllllllllllllllllll}569 & \text { S } & A & R & \text { L } & \text { A } & \text { S } & \text { V } & \text { C } & \text { L } & \text { W } & \text { I } & \text { L } & \text { V } & \text { F } & \text { G } & \text { C } & \text { K } & \text { F } & \text { T }\end{array}$ $\mathrm{E}$ 
ATCGTACTTTTTCCTCACGCAATCCTTCAAAAACCCCATCTTGGTCATGGTGGGCATGAA

$\begin{array}{llllllllllllllllllllll}589 & S & Y & F & F & L & T & Q & S & F & K & N & P & I & L & V & M & V & G & M\end{array}$ $\mathrm{K}$

\section{1}

GATCCAGGGATGCAATGACAAGTACTTCGGAGACAATCTGTGTCGCAACCAGGCGGCGTT $\begin{array}{lllllllllllllllllllll}609 & I & Q & G & C & N & D & K & Y & F & G & D & N & L & C & R & N & Q & A & A \\ F\end{array}$

2361

CACGCTGACGATCATGTACCTGATGGATCTCGTTCTGTTCTTTTTGGACACCTTCTTGTG

$\begin{array}{lllllllllllllllllllll}629 & \text { T } & \text { L } & \text { T } & \text { I } & M & \text { Y } & \text { L } & M & \text { D } & \text { L } & \text { V } & \text { L } & \text { F } & \text { F } & \text { L } & \text { D } & \text { T } & \text { F } & \text { L }\end{array}$
W

\section{1}

GTGGATCATCTGGAATACTGTCTTTAGTATCGCCCGATCATTCATGCTTGGATTATCGAT $\begin{array}{llllllllllllllllllllll}649 & W & I & I & W & N & T & V & F & S & I & A & R & S & F & M & L & G & L & S\end{array}$ I

2481

ATGGACACCTTGGAAGGATATCTATACGCGCCTGCCTAAGCGAATTTACTCAAAGTTGCT $\begin{array}{lllllllllllllllllllll}669 & W & T & P & W & K & D & I & Y & T & R & L & P & K & R & I & Y & S & K & L\end{array}$ L

\section{1}

GGCGACCTCAGATATGGAGACAAAGTACAAGCCAAAGGTCAGTGAACCGTCTACAGATAG

$\begin{array}{llllllllllllll}689 & \text { A } & \text { T } & S & D & M & E & \text { T } & K & Y & K & P & K\end{array}$

2601

AGCCATCTAACGTGAACGTATTGCAGGTGCTGGTGTCTCAAATCTGGAACGCGATCATCA

701

$\begin{array}{lllllllllllllll}V & L & V & S & Q & I & W & N & A & I & I\end{array}$

2661

TCTCAATGTACCGTGAACACTTGCTCTCCATCGACCACGTGCAAAAGTTGTTGTATCATC

$\begin{array}{llllllllllllllllllllll}712 & \text { I } & S & M & Y & R & E & H & \text { L } & \text { L } & \text { S } & \text { I } & \text { D } & \text { H } & \text { V } & \text { Q } & \text { K } & \text { L } & \text { L } & \text { Y } & \text { H }\end{array}$ 2761

AGGTCGACGCTGGCCAAGACGGACGTCGCAGCCTTAGAGCACCTCCTTTCTTCATCTCGC

$\begin{array}{llllllllllllllllllllll}732 & Q & V & D & A & G & Q & D & G & R & R & S & L & R & A & P & P & F & F & I & S\end{array}$ 2821

AGAGCGACAAGGGCTTCAAGGGCGAGTTCTTCACTCCTGGCAGCGAAGCAGAGCGGCGGA

$\begin{array}{llllllllllllllllllllll}752 & Q & S & D & K & G & F & K & G & E & F & F & T & P & G & S & E & A & E & R & R\end{array}$ 2881

TATCATTCTTCGCGCAGTCGCTTACGACGGCTGTTCCGGAACCGTTACCCGTCGATGCGA

$\begin{array}{lllllllllllllllllllll}772 & I & S & F & F & A & Q & S & L & T & T & A & V & P & E & P & L & P & V & D & A\end{array}$ 2941

TGCCGACATTTACCGTGCTGACACCCCATTACAGCGAGAAGGTAAGCGGTACTCGTATTC

$\begin{array}{llllllllllllllll}792 & M & P & T & F & T & V & L & T & P & H & Y & S & E & K\end{array}$

3001

GTCTTGATGGAGGTTCATGTGTCCTTTGTAGATCCTGCTCTCGCTTAGGGAGATCATTAG 
AGAGGAGGACCAGAACACCCGAGTAACGTTGCTGGAATACCTCAAGCAGTTGCATCCTGT

$\begin{array}{llllllllllllllllllllll}816 & E & E & D & Q & N & T & R & V & T & L & L & E & Y & L & K & Q & L & H & P\end{array}$
$\mathrm{V}$

3121

CGAGTGGGATAATTTCGTCAAGGACACGAAGATTCTCGCGGAAGAGTCTGCTATGTACAA $\begin{array}{llllllllllllllllllllllllll}836 & E & W & D & N & F & V & K & D & T & K & I & L & A & E & E & S & A & M & Y\end{array}$ $\mathrm{N}$

\section{1}

CGGACCCAGTCCATTCGGCACTGACGAGAAAGGACAGTCCAAGACTGACGACCTACCGTT

$\begin{array}{lllllllllllllllllllll}856 & G & P & S & P & F & G & T & D & E & K & G & Q & S & K & T & D & D & L & P\end{array}$ $\mathrm{F}$

3241

CTACTGCATCGGCTTCAAGTCCGCCGCACCCGAGTTTACTCTTCGTACGCGTATATGGGC

$\begin{array}{lllllllllllllllllllll}876 & \text { Y } & C & \text { I } & \text { G } & \text { F } & \text { K } & \text { S } & \text { A } & \text { A } & \text { P } & \text { E } & \text { F } & \text { T } & \text { L } & \text { R } & \text { T } & \text { R } & \text { I } & \text { W }\end{array}$ A

3301

CTCGCTCCGTGCACAGACGCTCTATCGCACAGTATCGGGCATGATGAACTATTCGAAGGC

$\begin{array}{llllllllllllllllllllll}896 & S & L & R & A & Q & T & L & Y & R & T & V & S & G & M & M & N & Y & S & K\end{array}$ A

3361

GATCAAACTGTTGTACCGTGTTGAGAACCCCGAAGTCGTACAGCTCTTTGGAGGCAACAC

$\begin{array}{lllllllllllllllllllll}916 & I & K & L & L & Y & R & V & E & N & P & E & V & V & Q & L & F & G & G & N\end{array}$ $\mathrm{T}$

3421

CGACAAACTCGAGCGGGAACTCGAGCGTATGGCACGGCGCAAGTTCAAGTTTGTCGTCTC

$\begin{array}{lllllllllllllllllllll}936 & \mathrm{D} & \mathrm{K} & \mathrm{L} & \mathrm{E} & \mathrm{R} & \mathrm{E} & \mathrm{L} & \mathrm{E} & \mathrm{R} & \mathrm{M} & \mathrm{A} & \mathrm{R} & \mathrm{R} & \mathrm{K} & \mathrm{F} & \mathrm{K} & \mathrm{F} & \mathrm{V} & \mathrm{V}\end{array}$ $\mathrm{S}$

3481

TATGCAGCGGTACTCCAAGTTCAACCGGGAAGAACAAGAGAACGCCGAATTTCTACTTCG

$\begin{array}{lllllllllllllllllllll}956 & M & Q & R & Y & S & K & F & N & R & E & E & Q & E & N & A & E & F & L & L\end{array}$ $\mathrm{R}$

3541

TGCATATCCGGACCTGCAAATCGCGTATCTCGAAGAGGAACCCGCGCGCAAGGAGGGCGG

$\begin{array}{llllllllllllllllllllll}976 & A & Y & P & D & L & Q & I & A & Y & L & E & E & E & P & A & R & K & E & G\end{array}$ G

3601

TGACCCTCGTCTGTTCTCCGCGCTGATCGACGGCCACTCGGAGTTCATCAACGAAAGTGG

$\begin{array}{lllllllllllllllllllll}996 & D & P & R & L & F & S & A & L & I & D & G & H & S & E & F & I & N & E & S\end{array}$ G

3661

CCGCCGTCGACCCAAGTTCCGCATCGAGCTTCCTGGTAACCCCATTCTCGGAGACGGAAA

$\begin{array}{lllllllllllllllllllll}1016 & R & R & R & P & K & F & R & I & E & L & P & G & N & P & I & L & G & D & G\end{array}$ 
GTCCGACAACCAGAATCACGCTATCATTTTCTACCGCGGCGAGTATCTCCAGCTCATCGA $\begin{array}{lllllllllllllllllllll}1036 & \mathrm{~S} & \mathrm{D} & \mathrm{N} & \mathrm{Q} & \mathrm{N} & \mathrm{H} & \mathrm{A} & \mathrm{I} & \mathrm{I} & \mathrm{F} & \mathrm{Y} & \mathrm{R} & \mathrm{G} & \mathrm{E} & \mathrm{Y} & \mathrm{L} & \mathrm{Q} & \mathrm{L} & \mathrm{I}\end{array}$ $\mathrm{D}$

3781

TGCGAATCAGGACAACTACCTTGAGGAATGTCTCAAGATCCGTAACGTACTTGGCGAATT

$\begin{array}{llllllllllllllllllllllllll}1056 & A & N & Q & D & N & Y & L & E & E & C & L & K & I & R & N & V & L & G & E\end{array}$ $\mathrm{F}$

3841

CGAGGAGTACTCAGTCTCGAGCCAGAGCCCGTACGCACAATGGGGCCACAAGGACTTCAA $\begin{array}{lllllllllllllllllllll}1076 & E & E & Y & S & V & S & S & Q & S & P & Y & A & Q & W & G & H & K & D & F\end{array}$ $\mathrm{K}$

3901

GAAATCCCCCATTGCCATCGTCGGTGCGCGCGAGTATATCTTCTCGGAAAATATCGGCAT $\begin{array}{llllllllllllllllllllllllllll}1096 & K & S & P & I & A & I & V & G & A & R & E & Y & I & F & S & E & N & I & G\end{array}$ I

3961

TCTCGGTGATCTCGCGGCCGGAAAGGAACAGACATTCGGTACCCTCACCGCGCGGTCGTT

$\begin{array}{lllllllllllllllllllll}1116 & L & G & D & L & A & A & G & K & E & Q & T & F & G & T & L & T & A & R & S\end{array}$ L

4021

GGCATGGATTGGCGGCAAGCTGCATTATGGCCACCCCGATTTCCTCAACGCGCTGTACAT $\begin{array}{llllllllllllllllllllllllll}1136 & A & W & I & G & G & \text { K } & \text { L } & \text { H } & \text { Y } & \text { G } & \text { H } & \text { P } & \text { D } & \text { F } & \text { L } & \text { N } & \text { A } & \text { L } & \text { Y }\end{array}$ $\mathrm{M}$

4081

GACCACCCGTGGCGGTGTCTCGAAGGCGCAGAAGGGTCTGCACCTTAACGAGGATATCTA

$\begin{array}{lllllllllllllllllllll}1156 & T & T & R & G & G & V & S & K & A & Q & K & G & L & H & L & N & E & D & I\end{array}$ Y

4141

CGCTGGAATGACGGCGTTCGGGCGCGGAGGTCGCATCAAGCACACAGAGTACTATCAGTG $\begin{array}{lllllllllllllllllllll}1176 & A & G & M & T & A & F & G & R & G & G & R & I & K & H & T & E & Y & Y & Q\end{array}$ C

4201

CGGTAAAGGTCGCGACCTCGGCTTTGGCACGATCCTCAACTTCCAGACGAAGATCGGCAC $\begin{array}{lllllllllllllllllllll}1196 & G & K & G & R & D & L & G & F & G & T & I & L & N & F & Q & T & K & I & G\end{array}$ T

4261

GGGTATGGGCGAGCAGATGCTCAGTCGGGAATACTACTACCTCGGCACGCAGCTCCCGAT

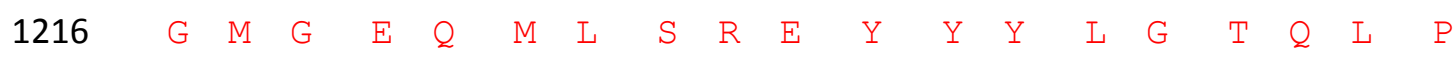
I

4321

TGATCGTTTCCTCACTTTCTACTATGGGCATCCTGGGTTCCACATCAATAACATGCTTGT 1236 $\begin{array}{llllllllllllllllllll}\text { D } & R & \text { F } & \text { L } & \text { T } & \text { F } & \text { Y } & \text { Y } & \text { G } & \text { H } & \text { P } & \text { G } & \text { F } & \text { H } & \text { I } & \text { N } & \text { N } & \text { M } & \text { L }\end{array}$ 


\section{1}

CATCCTGTCCGTTCAGATATTCATTGTGACGAGTAAGTGTTATGTGTTATTTGTGCTCTG $\begin{array}{lllllllllllllll}1256 & I & L & S & V & \varrho & I & F & I & V & T\end{array}$

4441

CATGGTGCTTATGAATTTGATTCGCAGTGGTTTTCTTGGGGACGTTGAACGACCAGCTGC
1266
$\begin{array}{llllllllllllllll}\text { M } & \text { V } & \text { F } & \text { L } & \text { G } & \text { T } & \text { L } & \text { N } & \text { D } & \text { Q } & \text { L }\end{array}$
4501

TCGTCTGCAAGTATTCCTCTTCGGGCCAGTTTATCGGTACAACAGGATGCTACAATCTCA

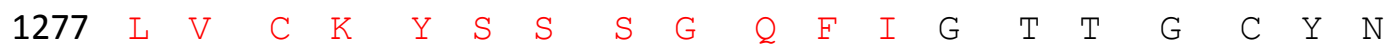
L

4561

CTCCTGCGTTCCAGTGGATCGACCATTGCATCATCAGTATTTTCTTGGTATTTATGATTG $\begin{array}{llllllllllllllllllll}1297 & \mathrm{~T} & \mathrm{P} & \mathrm{A} & \mathrm{F} & \mathrm{Q} & \mathrm{W} & \mathrm{I} & \mathrm{D} & \mathrm{H} & \mathrm{C} & \mathrm{I} & \mathrm{I} & \mathrm{S} & \mathrm{I} & \mathrm{F} & \mathrm{L} & \mathrm{V} & \mathrm{F} & \mathrm{M}\end{array}$ I

4621

CATACCTCCCCCTGTTCCTACAAGGTACGTCGAAGTGTCATGATTATAACGTCCAATTCG $\begin{array}{lllllllll}1317 & \text { A } & Y & \text { L } & \text { P } & \text { L } & \text { F } & \text { L } & Q\end{array}$

4681

ATAACCGTAATTCCGCAGAGCTTGTTGAGCGTGGAACTGGCAAAGCTGTATTTCGACTGG
1325
$\begin{array}{lllllllllllllll}\text { E } & \text { L } & \text { V } & E & \text { R } & G & \text { T } & G & K & \text { A } & V & \text { F } & \text { R } & \text { L }\end{array}$

4741

CGAAGCACTTCGGTTCGGCGTCTCCTGCGTTCGAAGTGTTCTCGACACAGATCTCTTCTC $\begin{array}{lllllllllllllllllllllllllll}1339 & \text { A } & K & H & F & G & S & A & S & P & A & F & E & V & F & S & T & Q & I & S & S\end{array}$ 4801

ATTCAATTATCACCAACTTGACTTTCGGAGGTGCAAGATACATCGCCACCGGCCGTGGCT $\begin{array}{lllllllllllllllllllll}1359 & \mathrm{H} & \mathrm{S} & \mathrm{I} & \mathrm{I} & \mathrm{T} & \mathrm{N} & \mathrm{L} & \mathrm{T} & \mathrm{F} & \mathrm{G} & \mathrm{G} & \mathrm{A} & \mathrm{R} & \mathrm{Y} & \mathrm{I} & \mathrm{A} & \mathrm{T} & \mathrm{G} & \mathrm{R}\end{array}$ G

\section{1}

TCGCGACGACGAGAATTTCATTTAGCATCCTATACTCGAGATTTGCGGGGCCAAGTATCT $\begin{array}{llllllllllllllllllll}1379 & F & A & T & T & R & I & S & F & S & I & L & Y & S & R & F & A & G & P & S\end{array}$ I

4921

ATCTCGGGATGAGGACATTGATTATGTTGTTATATGTGACTCTGACCATCTGGACGGGTT $\begin{array}{lllllllllllllllllllll}1399 & Y & L & G & M & R & T & L & I & M & L & L & Y & V & T & L & T & I & W & T\end{array}$ G

\section{1}

GGGTGACCTACTTCTGGGTATCCATCCTCGCTTTGTGCATTTCGCCGTTCCTCTTCAATC $\begin{array}{lllllllllllllllllllll}1419 & W & \text { V } & \text { T } & \text { Y } & \text { F } & \text { W } & \text { V } & \text { S } & \text { I } & \text { L } & \text { A } & \text { L } & \text { C } & \text { I } & \text { S } & \text { P } & \text { F } & \text { L } & \text { F }\end{array}$ $\mathrm{N}$

\section{1}

CCCATCAGTTCTCTTTTGCGGACTTTATCATTGACtACAGGTAAGCTAGAATTGTAGACC $\begin{array}{llllllllllllllll}1439 & \mathrm{P} & \mathrm{H} & \mathrm{Q} & \mathrm{F} & \mathrm{S} & \mathrm{F} & \mathrm{A} & \mathrm{D} & \mathrm{F} & \mathrm{I} & \mathrm{I} & \mathrm{D} & \mathrm{Y} & \mathrm{R}\end{array}$ 5101 
TAATCGTTCACGTCACTAACTTGAATTCCAGGGAGTTCTTGCGGTGGATGAACCGCGGCA

1453

$\begin{array}{llllllllll}E & F & L & R & W & M & N & R & G\end{array}$

5161

ACTCGCGCGTACACCAGAACTCTTGGATTGGCTATTGCCGGTTATCACGGACTATGATTA

$\begin{array}{lllllllllllllllllllllll}1462 & N & S & R & V & H & Q & N & S & W & I & G & Y & C & R & L & S & R & T & M & I\end{array}$ 5221

CAGGCTACAAGAAGAAGAGGCTCGGGCATCCTTCAGAGAAATTGTCGGGTGACGTCCCGCG

$\begin{array}{lllllllllllllllllllll}1482 & T & G & Y & K & K & K & R & L & G & H & P & S & E & K & L & S & G & D & V & P\end{array}$ $\mathrm{R}$

5282

TGCGGGCTGGCGAGCGGTCTTGTTCTCGGAGGTCATCTTCCCCATCGTGATGGCCACCCT

$\begin{array}{llllllllllllllllllllllllllll}1503 & \text { A } & G & \text { W } & \text { R } & \text { A } & \text { V } & \text { L } & \text { F } & \text { S } & \text { E } & \text { V } & \text { I } & \text { F } & \text { P } & \text { I } & \text { V } & \text { M } & \text { A } & \text { T }\end{array}$ L

5342

GTTCGTCATCGCATACATGCTCGTGAAGTCGTTCCCGGACAAGGATGGGAAGCAACCGCC

$\begin{array}{llllllllllllllllllll}1523 & F & V & I & A & Y & M & \text { L } & \text { V } & \text { K } & \text { S } & \text { F } & \text { P } & \text { D } & \text { K } & \text { D } & \text { G } & K & Q & P\end{array}$ $\mathrm{P}$

5402

CAGCCCCCTCATTCGAATCGCAATCGTATCTCTTGGGCCGATTGTTTGGAATGCTGCGAT

$\begin{array}{llllllllllllllllllllllll}1543 & \mathrm{~S} & \mathrm{P} & \mathrm{L} & \mathrm{I} & \mathrm{R} & \mathrm{I} & \mathrm{A} & \mathrm{I} & \mathrm{V} & \mathrm{S} & \mathrm{L} & \mathrm{G} & \mathrm{P} & \mathrm{I} & \mathrm{V} & \mathrm{W} & \mathrm{N} & \mathrm{A} & \mathrm{A}\end{array}$ I

5462

ATTGCTCGTCCTGTTCATGTTCTCGCTTTTCCTTGGACCGATGCTCGACACACCGTTCCC

$\begin{array}{llllllllllllllllllll}1563 & \text { L } & \text { L } & \text { V } & \text { L } & \text { F } & \text { M } & \text { F } & \text { S } & \text { L } & \text { F } & \text { L } & \text { G } & \text { P } & \text { M } & \text { L } & \text { D } & \text { T } & \text { P } & \text { F }\end{array}$ $\mathrm{P}$

5522

CAAGTTCGGATCATTGATGGCTTTCCTTGGGCACTCGCTCGGTGTCATCGGCATGATCGC

$\begin{array}{llllllllllllllllllllll}1583 & K & F & G & S & L & M & A & F & L & G & H & S & L & G & V & I & G & M & I\end{array}$ A

5582

ATTCTTCGAGTTCCTCGTAAGTCGTCTCACCTTATCTATTTCTTGACATCGCTCACTTTC

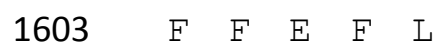

5642

CGCGGCACATAGTGGTTCCTCGAGCTGTGGAACGTCGCACACGCCGTGCTCGGTCTGATC $\begin{array}{llllllllllllllll}1608 & W & F & \text { L } & E & \text { L } & \text { W } & \text { N } & \text { V } & \text { A } & \text { H } & \text { A } & \text { V } & \text { L } & \text { G } & \text { L }\end{array}$ I

5702

GCAGTCATCTTCATTCAGCGCGCCATCCATAAGGTCCTGATTTCGGTGTTCCTGTCGCGC $\begin{array}{lllllllllllllllllllll}1624 & A & V & I & F & I & Q & R & A & I & H & K & V & L & I & S & V & F & L & S\end{array}$ $\mathrm{R}$

5762

GAGTTCAAGCATGACGAGACGAACCGGGCGTGGTGGACAGGTAAATGGTACGGCCGCGGG $\begin{array}{llllllllllllllllllll}1644 & \mathrm{E} & \mathrm{F} & \mathrm{K} & \mathrm{H} & \mathrm{D} & \mathrm{E} & \mathrm{T} & \mathrm{N} & \mathrm{R} & \mathrm{A} & \mathrm{W} & \mathrm{W} & \mathrm{T} & \mathrm{G} & \mathrm{K} & \mathrm{W} & \mathrm{Y} & \mathrm{G} & \mathrm{R}\end{array}$ G 


\section{2}

CTGGGCTCGCACGCGATGTCACAGCCTGCACGAGAGTTCATCGTGAAGATCATCGAGCTG

$\begin{array}{llllllllllllllllllll}1664 & \mathrm{~L} & \mathrm{G} & \mathrm{S} & \mathrm{H} & \mathrm{A} & \mathrm{M} & \mathrm{S} & \mathrm{Q} & \mathrm{P} & \mathrm{A} & \mathrm{R} & \mathrm{E} & \mathrm{F} & \mathrm{I} & \mathrm{V} & \mathrm{K} & \mathrm{I} & \mathrm{I} & \mathrm{E}\end{array}$ $\mathrm{L}$

5882

TCGCTGTGGAGCTCAGATTTCCTCACTGGCCACCTATTGCTCTTCTTGTTGACACCGCCA

$\begin{array}{llllllllllllllllllll}1684 & S & L & W & S & S & D & F & L & \text { T } & G & \text { H } & \text { L } & \text { L } & \text { L } & \text { F } & \text { L } & \text { L } & \text { T } & \text { P }\end{array}$ $\mathrm{P}$

5942

ATCCTCATACCGTACTTCGACCGGCTACACTCGACGATGCTCTGTGAGTAGCTATGCTAT

$\begin{array}{lllllllllllllll}1704 & \mathrm{I} & \mathrm{L} & \mathrm{I} & \mathrm{P} & \mathrm{Y} & \mathrm{F} & \mathrm{D} & \mathrm{R} & \mathrm{L} & \mathrm{H} & \mathrm{S} & \mathrm{T} & \mathrm{M} & \mathrm{L}\end{array}$

6002

GATtTATGCTAATGATGTACTGATATCGAGTCTGCAGTCTGGTTGAGGCCGTCAAAACA

1718

$\begin{array}{llllllll} & W & \mathrm{~L} & \mathrm{R} & \mathrm{P} & \mathrm{S} & \mathrm{K}\end{array}$

Q

6062

AATCCGCGCACCGTTGTATTCCATCAAGCAGAAGCGGCAACGTCGGTGGATTGTCATCAA

$\begin{array}{llllllllllllllllllll}1726 & I & R & A & P & L & Y & S & I & K & Q & K & R & Q & R & R & W & I & V & I\end{array}$ $\mathrm{K}$

6122

GTATGGTGCTGTCTACGTGCTCGCGATCGGCATCTTCGTTGCCCTCATCGTCATCCGTGG

$\begin{array}{lllllllllllllllllll}1746 & Y & G & A & V & Y & V & \text { L } & A & I & G & I & F & V & A & \text { L } & I & V & I\end{array}$

6182

GTATTGCTCATCTGTATGGTGTCATTGTTGCTCATCCCGCCCATGTAGCGCTCGTCTTCC

1764

P L V

$\mathrm{F}$

6242 GGCAATCGCTGACCTTCAACTGCAGCATTTGCCAACAGGTCTGA

$\begin{array}{lllllllllllllllllllllllll}1768 & R & Q & S & L & T & F & N & C & S & I & C & Q & Q & V & *\end{array}$

Figure S2 Nucleotide sequence and deduced amino acid sequence of putative glucan synthase in G. frondosa strain GF9801. 
Gf 1,3-beta-glucan svuthases $\mathrm{Pb}$ 1, 3-beta-glucan syuthases Tv 1,3-beta-glucan sunthases Gl 1,3-beta-glucan synthases Ds 1,3-beta glucan syuthases Lt 1,3-beta-glucan syuthases Or 1,3-beta-glucan syuthases Gt 1,3-beta-glucan syuthases Ag 1, 3-beta glucan syuthases

Gf 1, 3-beta-glucan stuthases $\mathrm{Pb}$ 1, 3-beta-glucan synthases Tv 1,3-beta-glucan suthases Gl 1,3-beta-glucan syuthases Ds 1,3-beta glucan syuthases Lt 1,3-beta-glucan syuthases Or 1, 3-beta-glucan syuthases Gt 1,3-beta-glucan syuthases Ag 1,3-beta glucan syuthases

Gf 1,3-beta-glucan svuthases $\mathrm{Pb}$ 1, 3-beta-glucan syuthases Tv 1,3-beta-glucan sunthases Gl 1,3-beta-glucan sputhases Ds 1,3-beta glucan syuthases Lt 1,3-beta-glucan syuthases Or 1,3-beta-glucan synthases Gt 1,3-beta-glucan synthases Ag 1,3-beta glucan syuthases

Gf 1,3-beta-glucan swuthases $\mathrm{Pb}$ 1, 3-beta-glucan sunthases Tv 1,3-beta-glucan sunthases Gl 1,3-beta-glducan synthases Ds 1,3-beta glucan syuthases Lt 1, 3-beta-glucan syuthases Or 1,3-beta-glucan synthases Gt 1,3-beta-glucan synthases Ag 1,3-beta glucan syuthases

Gf 1,3-beta-glucan sinthases $\mathrm{Pb}$ 1, 3-beta-glucan synthases Tv 1,3-beta-glucan sunthases Gl 1, 3-beta-glucan syutbases Ds 1,3-beta glucan syuthases Lt 1, 3-beta-glucan synthases Or 1, 3-beta-glucan synthases Gt 1,3-beta-glucan syuthases Ag 1,3-beta ellucan syuthases

Gf 1,3-beta-glucan swuthases $\mathrm{Pb}$ 1, 3-beta-glucan șuthases Tv 1,3-beta-glucan sunthases Gl 1,3-beta-glucan syuthases Ds 1,3-beta glucan syuthases Lt 1, 3-beta-glucan synthases Or 1,3-beta-glucan syuthases Gt 1, 3-beta-glucan syuthases Ag 1,3-beta glucan syuthases
MTGRSGPRSQEGMYVSSS-PS--SDPHTPFSHVHRQMPEPQRYYDKDSDQIDHYGR-RDT MTSRPGPHSQEGMYVSSSPPS--SDPHDPFHQPPMQMPDP-RYYDNDSEHLDRYDRHRET -MAGRGPRSQEGMYTSPSPPS--SDPHDFFSTTQMPEP-H-RYYDQEDDHDDQYGR-RDT MASRHGHHSQDGVYYSSSPS--SDPHPFHFPVQMPEPH-RYYDHDSDQLDRYDRQRDT MASRPGPRSQEGMYVPSSPPS--SDPHDFFHPVQMPEPH-RYYDHDSE---RYDRQRDT MARRPGPHSQEGMYVSSSPPS--SDPHDPFHQ-SMQMPDP-RYYDHDSEHLDRYDRHRDT MAGRPGHRSQEGVISSSPGS--SDPHDPFSHAQ--ARDPGRYYDHDS--VEHYTERRDT - MATPR-HFDHI-YPGSSPP---SDPHDFAS-----SHQRYYDKDSDQADPYRR-RDT MSGRPGHASQEG-YPSSSPSSLDAHQHDPFSP------PQRRYYDKDSE---DYGR-RDT

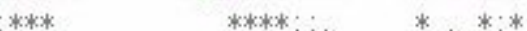

YGSDGSHGG-HDDDRYYDHAGAYDPYGPQPDTDSDVDVYGQKYAPSAESLGPPRVGVSES YASDGSHG--DDDRYYEQGGGIDY--PQPDTDSDVVVIGKQYPPSQESLGPPRIGISES YGSEGSHL---DEDRYYEQGGAYDY--AQQDTDSDVDVYGQKYPPSQESLGPPRMGISES YASDGSQA---DDDRYYEQGGTYDY--TMPDTDSDADVYGQKYVPSTESLGPPRMGISES YASDGSQG---DDDRYYEHGGAYDY--TQPDTDSDMDYYGQKYVPSTESLGPPRMGISES YASDGSMA---DDDRYYEHGGGYDY--AQPDTDSDVDVIGQKYIPSQESLGQPRMGISES YASDGSHAGLRDDDYYDHGGGIDY--ARPDTDSEADVYEPKY APSAESLGPPRLGISES YASDSSDARLKEDDRYYDKHGNYGRYGH-PDTDSDVDVYGQKYAPSAESLGPPRME---S YASDHS-----NTGFYDQHGAYDPYPP-QDTDSDGDAYGPRYAPSAESLGVPRMGISES

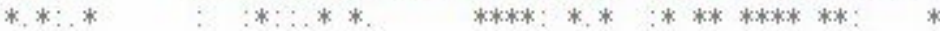

STPTFIDHLIGS-GGREPYPAHSSERQIPLSKEEIEDIFLDLTQKFGFQRDSMRNMFDFTM STPTFVDHLGV-SGREPYPAWSSERQIPLSKEEIEDIFLDLTQKFGFQRDSMRINFDFVM STPTFIDHLGY-SGREPYPAW'SSERQIPLSKEEIEDIFLDLTQKFGFQRDSMRAMFDFVM STPTFVDTHG---VREPYPAWSSERQIPLSKEEIEDIFLDLTQKFGFQRDSMRWMFDFVM STPTFVDHUGV-SVREPYPAWSSERQIPLSKEEIEDVFLDLTQKFGFQRDSMRRMFDFVM STPTFIDHLGV-SVREPYPAWISSERQIPLSKEEIEDIFLDLTQKFGFQRDSMRHMFDFVM STPTFVDSSG--GLREPYPAWSSERQIPLSKEEIEDIFLDLTQKFGFQRDSMRIMFDFVM STPTFVDYGGPPGAREQYPAHTSERSIPLSKEEIEDIFLDLTQKFGFQRDSMRAMFDFVM STPTFIDYGQ--GGRDAYP AN'WSERQIPLSKEEIEDIFLDLTQKFGFQRDSMRHMFDFLM

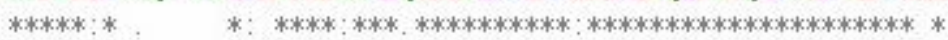

QLIDSRASRMSPHQALLTLHADYGGQHARYRKWYF ARQLDLDDAIGQTQNPGLRS---QLDDSRASFMSP FQALLTLHEDYIGGQHAWY RKWYF ARQLDLDDAIGSQQRPGLQRLRSQ QLIDSRASRMSP FQALLTLHADYIGGQHAWYRKWYF ARQLDLDDAIGQTQHPGLSRLRSQ QLIDSRASRMSPHQ ALLTLHADYIGGQHАWYRKWYF АНQLDLDDIVGSTQNPGLRRLRSQ QLIDSRASRMSP FQ ALLTLHADYIGGQHคWYRKWYF ARQDDLDDAIGSTQRPGLRRLRSQ QLIDSRASRMSP HQALLTLHADYIGGQHANYRKWYF AAQLDLDDAIGHTQRPGLQRLRSQ QQLDSRASRMSP FQALLTLHADYIGGQHAHYRKWYFAAQDDLDDAVGQTQRPGLKRLKSV QLIDSRASRMSPDQALLTLHADYIGGQHAWYRKWYF ARQLDLDDAVGQTQRPGLQRLKST QLIDSRASRMSPYQALLTLHADYIGGHHARYRKWYF AAQLDLDDAVGQQTQNPGLTRLKSK

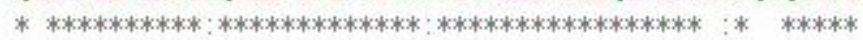

-TKRKGHKAVEKSLRIALDRWRQAMMIMSQYDRMRQIALYLUCWGEAAQVRFVPCLCFI KRKSKGYKKARERQLSSALDRWRQAMINMSQYDRMRQIALYLLLWGEAAQVRFVPECLCFI

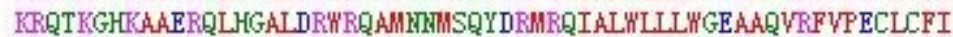

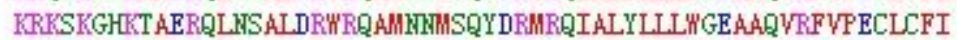

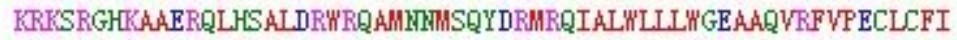
KRKSKGHKAAERQLLSALDRW'RQAMINMSQYDFMRQIALYLLW'GEAAQVRFVPECLCFI

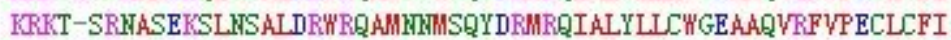
KRR-GGRG-AEISLSSAMERW'RQAMRIMSQYDRLRQIALYLCCWGEARQVRFMPECLCFI RGS-GKRPPHEISLNT AIERWRQAMIIMSQYDRLRQIALYLIUWGAHAVRFVPECLCFI

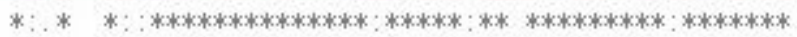

FKCADDYYRSPECQSRIDPVPEGLYLHAVIKPLYRFIRDQGYEVDGKFVRREKDHDTII FKCADDYYRSPECQHRVDPVPEGLYL HAVIKPLYRFIRDQGYEVVDGKFVRKEKDHEDII FKCADDYYRSPECQSRVDPVPEGLYLHAVVKPLYRFIRDQGYEVIDGKFVRKERDHDQII FKCADDYYRSPECQHEIDFVPEGLYLHAVVKPLYRFIRDQGYEVVEGKFVRKERDHDQII FKCADDYYRSPECQNRIDPVPEGLYLHAYVKFLYRFIRDQGYEVVDGKFVKEKDHQII FKCADDYYRSPECQSRVDPVPEGLYLHAVIKPLYRFIRDQGYEVVDGKFVRRERDHAEII FKCADDYYRSPECQSRVDPVPEGLYLRSVIKPLYRFIRDQGYEVVDGKFVRREKDHTII FKCADDYYRSPECQHRLDPVPEGLYLRAVIKPLYRFIRDQGYEVVDGKFVRRERDHWKI FKCADDYYRSPECQHEMEPVPEGLYLRAVIKPLYRFIRDQGYEVDGKFVRRERDHEDVI

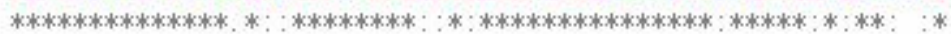

56

57

54

57

54

56

54

47

49

115

112

109

112

109

111

112

103

102

174

171

168

169

168

170

170

163

160

230

231

228

229

228

230

230

223

220

289

291

288

289

288

290

289

281

279

349

351

348

349

348

350

349

341

339 
Gf 1,3-beta-glucan stuthases $\mathrm{Pb} 1,3$-beta-glucan syuthases Tv 1,3-beta-glucan swuthases Gl 1,3-beta-glucan syuthases Ds 1,3-beta glucan syuthases Lt 1,3-beta-glucan synthases Or 1,3-beta-glucan synthases Gt 1,3-beta-glucan syuthases Ag 1,3-beta-glucan syuthases

Gf 1,3-beta-glucan sunthases $\mathrm{Pb} 1$, 3-beta-glucan syuthases Tv 1,3-beta-glucan suthases Gl 1,3-beta-glucan syuthases Ds 1,3-beta glucan synthases Lt 1,3-beta-glucan synthases Or 1,3-beta-glucan synthases Gt 1,3-beta-glucan synthases Ag 1,3-beta-glucan synthases

Gf 1,3-beta-glucan swnthases $\mathrm{Pb}$ 1, 3-beta-glucan syuthases Tv 1,3-beta-glucan snithases G1 1,3-beta-glucan syuthases Ds 1,3-beta-glucan synthases Lt 1,3-beta-glucan synthases Or 1,3-beta-glucan synthases Gt 1,3-beta-glucan syuthases Ag 1,3-beta glucan synthases

Gf 1,3-beta-glucan svuthases $\mathrm{Pb}$ 1, 3-beta-glucan syuthases Tv 1,3-beta-glucan svuthases G1 1,3-beta-glucan syuthases Ds 1,3-beta glucan synthases Lt 1,3-beta-glucan synthases Or 1,3-beta-glucan syuthases Gt 1,3-beta-glucan synthases Ag 1,3-beta glucan synthases

Gf 1,3-beta-glucan svuthases $\mathrm{Pb}$ 1, 3-beta-glucan synthases Tv 1,3-beta-glucan sunthases G1 1,3-beta-giducan syuthases Ds 1,3-beta glucan synthases Lt 1,3-beta-glucan syuthases Or 1,3-beta-glucan synthases Gt 1,3-beta-glucan syuthases Ag 1,3-beta glucan synthases

Gf 1,3-beta-glucan svuthases $\mathrm{Pb}$ 1, 3-beta-glucan synthases Tv 1,3-beta-glucan sunthases G1 1,3-beta-glucan synthases Ds 1,3-beta glucan syuthases Lt 1,3-beta-glucan synthases Or 1,3-beta-glucan syuthases Gt 1,3-beta-glucan synthases Ag 1,3-beta-glucrun synthases
GIDDVRQLFWYPEGIARTVLADKTRLVDLAPAQRFMKFDRID"WRAFFK-------GYDDVNQLFWYPEGIARIVLTDKTRLVDLPPSQRFMKFDRIDWHRVFFTYYEKRSFGHL GIDDVRQFWYPEGIARTVLTDKSRLVDLPPAQRFMKFDRVDWWRVFFKTYEKRSFGHL

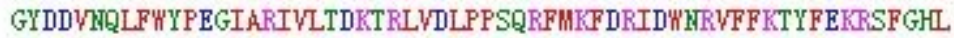
GYDDVMQLFWYPEGIARTVLTDKTRLVDLPPSQRFMKFDRID"KRVFFKYYFEKRFGHL GYDDVRQLFWYPEGARTVLTDKTRLVDLPPSQRFMKFDRID"WRVFFTYFEKRSFGHL GYDDVNQLFWYPEGIARIVLVDKTRLIDLPPARRFMKFDRID"NRAFFKTYYEKRSFGHL GYDDVNQLFWYPEIARTVLDDTRLVDVPFAQRFMKFDRVD"WRVFFKYYEKRSFGHL GYDDVIQLFWYPEGIARTVLTDKTRLVDLAPARRFMKFDRTD"WRAFFKTYYERRSFGHL

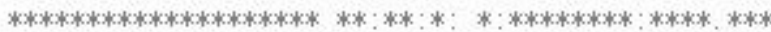

----TINVIHVSMYWYYTAYKSPTVYH--GIRST AMRHSATALGGAVATVIMILATLAE

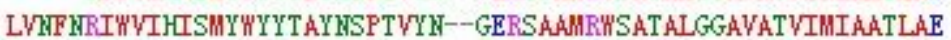
LVNFWRI'WIHISMYWFYTAYKSPTIYH--GEAHAAMRWSATALGGAVASIIMICATLAE

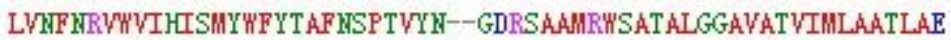
LVNFWRTWYHYSMYWFYTAYHSPTIYH--GDKSSAMRWSATALGGAVATIIMIAATLAE

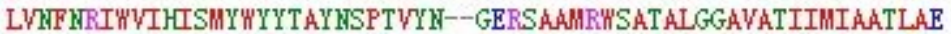
LVNFKRIWVIHСАM"WFYTAYNSPNIYH--GGKSAALSWSATALGGAVATIIMICATLAE LVNFWRIWVIHАMYWFYTAYNSPTVG--GDKSAнMSWSATALGGAVATTIMILATIAE LVRFRVYVIHISLYFYTAYKSPTVYQVHGQWVPALWSATALGGAVATVIMILATLTE

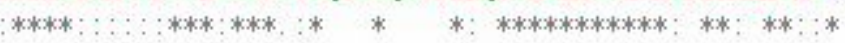

FSYIPTT"WHTSHLTRRLIFLAITLALTAGPTFYIAIAESSSPGGSLALILGIVQFFISV FSYIPTTWKRTSHLTRRLIFLIITLALTAGPTFYIAIAEKSSPGGSLALILGIVQFFISV FSYIPTTWHTSHLTRRLIFLFVTLALTAGPTFYIAIAESHSPGGSLALILGIVQFFIAV FSYIPTTWHKTSHLTRRLLFLAVTLALTAGPTFYIAIAESHSPGGSLALILGIVQFFISV FSYIPTTWKNTSHLTRRLIFLVITLALTAGPTFYIAIAESHSPGGSLALILGIVQFFISV FSYIPTTWHKTSHLTRRLFLAITLALTAGPTFYIAIAESSSPGGSLALILGIVQFFISV FSYIPTTWHTSHLTRLIFLLVTLALTAGPTFYIAIAETHSPGGSLALILGIVQFFISA FSYIPTTWRTSSHTRRLIFLFITLGLTAGPTFYVAIAVRRSGGSLPLILGIVQFFISV FSYIPTTWHTSSHLTRRLFLFVTLGLTCGPTFYVAIAEHHGSGGQLSLILGIVQFFISV

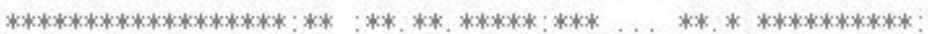

VATLLFAFLPSGRMFGDRVAGKSRKYLASQTFTASYPTLKASARLASVCLWILVFGCKFT VATLLFAILPSGRMFGDRVAGKSRKYLASQTFTGSYPTLKPSARTASLILWLLVFSCKFV VATLIFAILPSGRMFGDRVAGKSRKYLASQTFTASYPSLSSSARLASVGL"'LLVFGCKFT VATLLFAILPSGEMFGDRVAGKSRKYLASQTFTASYPSLKSSARMASVLL"'FLVFGCKFT VATLLFAVLPSGRMFGDVAGKSRKYLASQTFTASYPSLTSSARIASLFLWFLVFGCKLT VATLLFAILPSGRMFGDRVAGKSRKYLASQTFTASYPSLKFSARTASLILWVLVFGCKLT VATLLFAVLPSGRMFGDRVAGKSRKYLASQTFTASYPTLKSTARLASVTLWLLIF ACKFT VATLLFAIMPSGRMFGDRVAGKSRKYLASQTFTASYPDLLRARIASIALWVLIFGCKFV VATLLFAIMPSGRMFGDRVAGKSRKYLASQTFTASYPTLETAPRIASFLLWLLIFACKFV

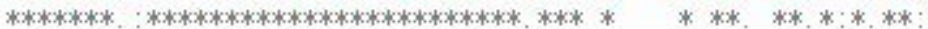

ESYFFLTQSFKAPILVMVGMKIQGCHDKYFGDRLCRHQАНFTLTIMYLMDLVLFFLDTFL ESYFFLTLSFKAPILVMVGMQVRRCSDKYFGHALCRHQAAFTLTIMYLMDLVLFFDTFL ESYFFLTLSFKRPIRVMVGMQIQGCKDKYFGHALRRQAAFTLTIMYLMDLVLFFLDTFL ESYFFLTLSFKMPIRVMVGMEIQGCHDKYFGIGLCRHQATFTLTIMYLMDLVLYFLDTFL ESYFFLTLSFKAPIRVMVGMQIQGCHDKYFGHALCRHQAAFTLTIMYLMDLVLYFLDTFL ESYFFLTLSFKASIRVMVGMQVKOCSDKYFGHALCRHQAAFTLAIMYVMDLVLYFLDTFL ESYFFLTLSFRMPIRAMVGMKVQHCHDLFGSALCRHQAVTLMFMHMDLILFFLDTFL ESYFFLTTSFSVSIRVMVGMKVQGCHDRYFGHALCRHQAнFTLTIMYIMDLVLFFLDTFL ESYFFLTLSFRDPIRVMVGMKIQGCHDRL"'GHALCTHQA AFTLTIMYVMDLALFFDTFL

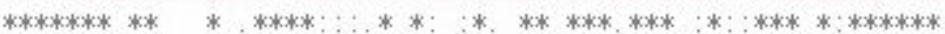

"WIINKTVFSIARSFMLGLSI"WPWKDIYTRLPKRIYSKLLATSDMETKYKPKVLVSQI" "WIIINKTVFSIGRSF ALGLSIWTPWKDIYTRLPKRIYSKILATADMETKYKPKVLVSQIW "W"WINWTVSIGRSFALGLSINTPWKDIYTRLPKRIYSKILATADMETKYKPKVLVSQIW "W'WIWWTVFSIGRSFGLGLSWTPWKDIYTRLPKRIYSKILATSDMETKYKPKVLVSQIW "WIIWHTVFSIGRSF ALGLSINTF"WDIYTRLPKRIYSSILAT ADMETKYKF KVLVSQIW "WIINHTVFSIGRSF ALGLSINTPWKDIYTRLPKRIYSKILATADMETKYKP KVLVSQIW" Y--------IGRSF ALGLSIWTPWKIDIFTRLPKRIYAKLLATSDMETKYKPKVLVSQIW' "YIINWTVSIARSFMLGLSIWTPWKDIYTRLPKRIYAKLLATADMEVKYKPKVLVSQIW" "YIIWNTVFSIGRSFSLGLSIWTPWKDIYTRLPKRIYAKLLATHDMEVKYKPKVLVSQIW" 
Gf 1,3-beta-glucan svinthases $\mathrm{Pb}$ 1,3-beta-glucan syuthases Tv 1,3-beta-glucan sunthases G1 1,3-beta-glucan synthases Ds 1, 3-beta glucan synthases Lt 1,3-beta-glucan synthases Or 1,3-beta-glucan synthases Gt 1, 3-beta-glucan syuthases Ag 1, 3-beta glucan syuthases

Gf 1,3-beta-glucan svuthases $\mathrm{Pb}$ 1, 3-beta-glucan syuthases Tv 1,3-beta-gucan sunthases GI 1,3-beta-glucan synthases Ds 1, 3-beta glucan syuthases Lt 1, 3-beta-glucan syuthases Or 1,3-beta-glucan synthases Gt 1,3-beta-glucan syuthases Ag 1, 3-beta -ylucan syuthases

Gf 1,3-beta-glucan sunthases $\mathrm{Pb}$ 1, 3-beta-glucan syuthases Tv 1,3-beta-glucan sunthases Gl 1,3-beta-glucan șuthases Ds 1,3-beta -glucan syuthases Lt 1,3-beta-glucan syuthases Or 1,3-beta-glucan synthases Gt 1,3-beta-glucan syuthases Ag 1,3-beta glucan syuthases

Gf 1,3-beta-glucan swuthases $\mathrm{Pb}$ 1,3-beta-glucan synthases Tv 1,3-beta-glucan sunthases Gl 1, 3-beta-glucan șnthases Ds 1,3-beta glucan syuthases Lt 1,3-beta-glucan syuthases Or 1,3-beta-glucan syuthases Gt 1,3-beta-glucan syuthases Ag 1,3-beta gluccan syuthases

Gf 1,3-beta-glucan swuthases $\mathrm{Pb}$ 1,3-beta-glucan sunthases Tv 1,3-beta-ducan sunthases Gl 1,3-beta-glucan syuthases Ds 1,3-beta glucan syuthases Lt 1, 3-beta-glucan syuthases Or 1,3-beta-glucan synthases Gt 1,3-beta-glucan synthases Ag 1,3-beta -lucau syuthases

Gf 1,3-beta-glucan swuthases $\mathrm{Pb}$ 1,3-beta-glucan syuthases Tv 1,3-beta-glucan suthases Gl 1,3-beta-glucan șuthases Ds 1,3-beta glucan syuthases Lt 1, 3-beta-glucan syuthases Or 1,3-beta-glucan syuthases Gt 1,3-beta-glucan synthases Ag 1, 3-beta glucan syuthases
HAIIISMYREHLISIDHVQKLLYHQVDAG--QDGRRSLRAPPFFISQSDKGFKGEFFTPG HAIIISMYREHLISIEHVQKLLYHQVDVG--QDGRRSLRAP AFFISQSDKGYRGQFFTPG HAIIISMYREHLISIEYVQKLLYHQVDVG--QDGRRSLRAPAFFISQSDKAFRGEFFTPG HAIIISMYREHLISIEHVQKLIYHQVDA---QDGRRSLRAPAFFIAQSDKGFRGQFFTPG HAIIISMYREHLISIEHVQKLIYHQVDA---QDGRRSLRAPAFFISQGDKGYRGQFFTPG HAIIISMYREHLISIEHVQKLIYHQVDYG--QDGRRSLRAP AFFISQSDKGFRGEFFTPG HAIIISMYREYLISINFVQKLLYKQVDVG--QDGRRSLRAPAFFISQSDKGLKGQFFTPG HAIIISMYREHLISIDHVQRLLYHQIDAG--GDGRRSLRAPPFFISQSDKGYKGEFFPRG HAIIISMYREYLISIDHVQKLIYHQVDGGPGAHGRRSLRAPPFFISQRDKGFKGEFFPKG

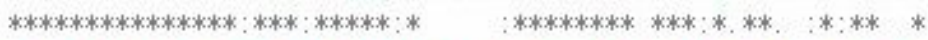

SEAERRISFFAQSLTTAVPEPLPVDAMPTFTVLTPHYSEKILISLREIIREEDQYTRVTL SEAERRISFF AQSLTTAVPEPLPVDAMPTFTVLTPHYSEKILISLREIIREEDQYTRVTL SEAERRISFF AQSLTTAVPEPLPVDAMPTFTILTPHYSEKILLSLREIIREEDQKTRVTL SEAERRISFF AQSLTTAVPEPLPVDAMPTFTVLTPHYSEKILLSLREIIREEDQNTRVTL SEAERRISFF AQSLTT AVPEPLPVDAMPTFTVLTPHYSEKILLSLREIIREEDQNTRVTL SEAERRISFF \&QSLTT AVPEPLPVDAMPTFTVLTPHYSEKILISLREIIREEDQNTRVTL SEAERRISFF AQSLTTAVPEPLPVDAMPTFTVLTPHYSEKILISLREIIREEDQNTRVTL SEAERRISFF ADSLTT ALPEPVPVDAMPTFTVLTPHYSEKILISLREIIREEDQTTRVTL SEAERRISFF AQSLTT AMPEPLPVDAMPTFTVLTPHYSEKILISLREIIREEDKMTRVTL

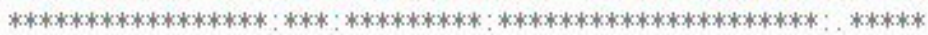

LEYLKQLLPVEWDKFVKDTKILAEESAMYHGPSPFG-TDEKGQSKTDDLPFYCIGFKSA A LEYLKQLFPVEWDHFVKDTKILAEESQMYYGSKPFG-MDEKGQSKADDLPFYCIGFKSA LEYLKQLUYPVEWDHFVKDTKILAEESQTFHGSSPFG GMDEKGASKADDLPFYCIGFKSA LEYLKQLFYPVWDIFVITTKILAEESQMYHGPSPFG-MDEKGQSKADDLPFYCIGFKSAA LEYLKQLLPVEW'DKFVKDTKILAEESQMYHGAKFFG--DEKGQSKTDDLPFYCIGFKSAA LEYLKQLFPVEWDHFVIDTKILAEESQMYYGAKPFG--DEKARSKADDLPFYCIGFKSAн LEYLKQLVPIEWDFFVKDTKILAEESAMYHGSHPFG-VDEKGQSKIDDLPFYCIGFKSАん LEYLKQLLYPVEWDNFVKDTKILAEESAMYHGP NPFG-ADEKGQSKTDDLPFYCIGFKSAA LEYLKQLHPIEWEYFVKDTKILAEESAMFHGTHPFG-DHEKARSKTDDLPFYFIGFKSA A

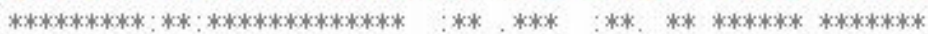

PEFTLRTRIW'ASLRARTLYRTVSGMMYYSKAIKILYRVENPEVVQFGGNTDKIERELER PEFTLRTRIW ASLRAQTLYRTVSGMMYYAKAIKILYRVENPEVYQLFGGKTDKIERELER PEFTLRTRIW'ASLRAQTLYRTVSGMMNISKAIKILYRVENPEVVQLFGGKTDKIERELER PEFTLRTRIN'ASLRARTLYRTVSGMMFY AKATKLLYRVERPEVVQLFGGNTDKLERELER

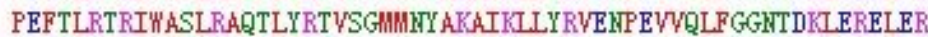
PEFTLRTRIW ASLRAQTLYRTVSGMMIYAKAIKLLYRVENPEVYQLFG GYTDKLERELER PEFTLRTRIN'ASLRAQTLYRTVSGMMYYAKAIKLLYRVERPEVVQLFGGITDKLERELER PEFTLRTRIN'ASLRAQTLYRTVS GMMIYSKAIKLLYRVENPEVVQLFGGKTDKIERELER PEFTLRTRIW'ASLRAQTLYRTYSGMMIYSKAIKLLYRVENPEVYQMFGGNTDKLERELER

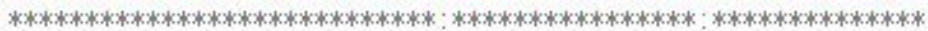

MARRKFKFWSMQRYSKFHREEQEHAEFLLRAYPDLQIAYLEEEPARKEGGDPRLFSALI MARRKFIFYYSMQRYSKFWREEQENAEFLLRAYPDLQIAYLEEEPPRKEGGDPRLFSALI MARRKFIFYYSMQRYSKFHREEQENAEFLLRAYPDLOIAYLEEEPPRKEGGDSRIFSALI MARRKFKFYVSMQRYSKFHREEQEHAEFLLRAYPDLQIAYLEEEPFRIEGGDPRLFSALI MARRKF KFYVSMQRYAKFHREEQENAEFLLRAYPDLQIAYLEEEPPRKEGGDPRLFSCLI MARRKFIFYVSMQRYSKFHREEQENAEFLLRAYPDLQIAYLEEEPPRKEGGDPRLFSALI MARRKFKLVISMQRRSKF NREEQENTEFLLCVYPDLOIAYLEEEPARKEGGDPRLFSALI MARRKFKFYVSMQRYSKFHKEEQEHAEFLLRAYPDLQIAYLDEEPPRKEGGDPRLFSALI MARRKFKFYVMQRYSKFWKEEQENAEFLLRAYPDLRIAYLEEEPPRKEGGDPRIFSALI

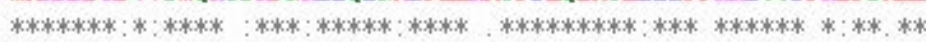

DGHSEFIKESGRRRPKFRIELPGHPILGDGKSDHQHHAIIFYRGEYLQLTDARDDNYLEE DGHSEFIADSGRRRPKFRIELPGKPILGDGISDHQHLAIIFYRGEYLQLLDDARQDWYLEE DGHSEFIADT GRRKPKFRIELPGNPILGDGISDHQHHAIIFYRGEYLQLIDAHQDWYLEE DGHSEFIAETGRRRPKFRIELPGKPILGDGKSDHQRHAIIFYRGEYLQLTDARQDNYLEE DGHSEFIPETSRRRPKFRIELPGNPILGDGKSDHQNHAIIFYRGEYLQLIDAHQDNYLEE DGHSEFIADTGRRRPKFRIELPGHPILGDGISDHQHUAIIFYRGEYLQLIDARDDWYLEE DGHSEFIPDTGRRRPKFRIELPGNPILGDGKSDHQNHAIIFY RGEYLQLIDAHQDNYLEE DGHSDFVPETGRRRPKFRIELPGNPILGDGKSDHQHYAIIFYRGEYLQLIDAHQDNYLEE DGHSEFIPETGRRKPKFRIELPGNPILGDGKSDHQNUALIFYRGEYLQLIDARQDNYLEE

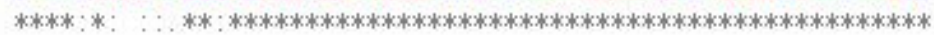

988

1006 1004

1003

1001

1004

995

996

998

1048

1066

1064

1063

1061

1064

1055

1056

1058 
Gf 1,3-beta-glucan svuthases $\mathrm{Pb}$ 1, 3-beta-glucan syuthases Tv 1,3-beta-glucan swnthases G1 1,3-beta-glucan synthases Ds 1,3-beta glucan syuthases Lt 1,3-beta-glucan synthases Or 1,3-beta-glucan syuthases Gt 1,3-beta-glucan synthases Ag 1,3-beta glucan syuthases

Gf 1,3-beta-glucan svuthases $\mathrm{Pb}$ 1, 3-beta-glucan syuthases Tv 1,3-beta-glucan sunthases G1 1,3-beta-glucan synthases Ds 1,3-beta glucan syuthases Lt 1,3-beta-glucan syuthases Or 1,3-beta-glucan syuthases Gt 1,3-beta-glucan synthases Ag 1,3-beta glucan synthases

Gf 1,3-beta-glucan svuthases $\mathrm{Pb}$ 1, 3-beta-glucan syutbases Tv 1,3-beta-glucan swuthases G1 1,3-beta-glucan syuthases Ds 1,3-beta glucan synthases Lt 1,3-beta-glucan synthases Or 1,3-beta-glucan synthases Gt 1,3-beta-glucan syuthases Ag 1,3-beta glucan syuthases

Gf 1,3-beta-glucan svuthases $\mathrm{Pb} 1$, 3-beta-glucan synthases Tv 1,3-beta-glucan sunthases G1 1,3-beta-giducan syuthases Ds 1,3-beta glucan synthases Lt 1,3-beta-glucan syuthases Or 1,3-beta-glucan syuthases Gt 1,3-beta-glucan syuthases Ag 1,3-beta glucan synthases

Gf 1,3-beta-glucan svuthases $\mathrm{Pb} 1,3$-beta-glucan synthases Tv 1,3-beta-glucan sunthases Gl 1,3-beta-gucan synthases Ds 1,3-beta-glucan synthases Lt 1,3-beta-glucan synthases Or 1,3-beta-glucan synthases Gt 1,3-beta-glucan synthases Ag 1,3-beta-glucan synthases

Gf 1,3-beta-glucan svuthases $\mathrm{Pb} 1$, 3-beta-glucan synthases Tv 1,3-beta-glucan swnthases G1 1,3-beta-giducan synthases Ds 1,3-beta-glucan synthases Lt 1,3-beta-glucan syuthases Or 1,3-beta-glucan syuthases Gt 1,3-beta-glucan syuthases Ag 1,3-beta glucan synthases
CLKIRNVLGEFEYSVSSQSPYAQW'GHITFKKSPIAIVGAREYIFSENIGILGDLAAGKE CLKIRHVLAEFEEYWYSSQSPYAQWGHKDFKKSPIATVGAREYIFSEHIGILGDLAAGKE CLKIRWVLAEFEYWVSSQSPYAQWGHKDFKKSPIAIVGAREYIFSENIGILGDLAAGKE CLKIRRVLAEFEEYSVSSQSPYAQWGHKTFKKSPIAIVGAREYIFSEKIGILGDLAAGKE CLKIRWVLAEFEEYAVSSQSPYAQWGHITFKKSPIAIVGAREYIFSENIGILGDLAAGKE CLKIRHVLAEFEEYWVSSQSPYAQW'GHKDFKKSPIAIVGAREYIFSEMIGILGDLAAGKE CLKIRWVLEFEEYSVSTQSPYAQWGHKEFKRSPVAVGAREYIFSENIGILGDLAAGKE CLKIRHVLEFEEYSVSTQSPYATWGQKEFRKPPVAIVGAREYIFSENIGILGDIAAGKE CLKIRRVLSEFEEYKISTQSPYAHW'GHKEFKRSPVAIVGAREYIFSEKIGILGDLAAGKE

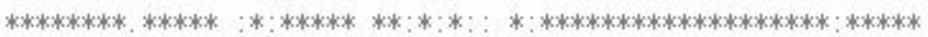

QTFGTLTARSLAWIGGKLHYGHPDFLKALYMTTRGGVSKAQKGLHLWEIYAGMTAFGRG QTFGTLAARSLAWIGGKLHYGHPDFLNALFMTTRGGVSKAQKGLHLNEDIY AGMHAFGRG

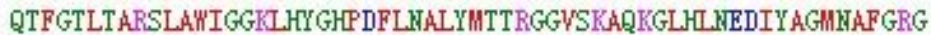

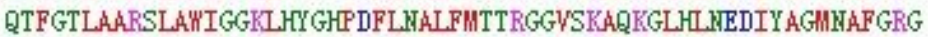

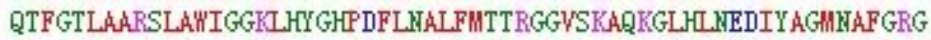
QTFGTLTARSLAWIGGKL_YYGHPDFLNALFMTTRGGVSKAQKGLHLसEDIYAGMNAFGRG QTFGTLTARSLAWIGGKL.HYGPDFLNAIFMTTRGGVSKARKGLHLNEDIYAGMHAFGRG

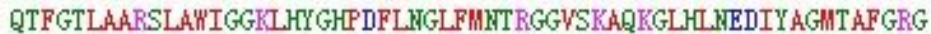

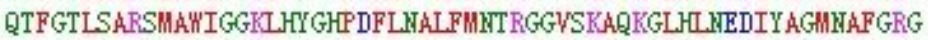

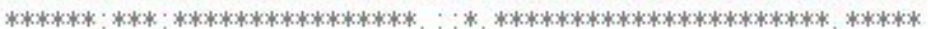

GRIKHTEYYQCGKGRDLGFGTILKFQTKIGTA-

GRIKHTEYYQCGKGRDLGFGTILKFQTKIGTGMGEQMLSREYYYLGTQLPIDRFLTFYYG GRIKHTEYYQCGKGRDLGFGTILNFQTKIGTGMGEQMLSREYYYLGTQLPVDRFLTFYYG GRIKHTEYYQCGKGRDLGFGTILKFTTKIGTGMGEQMLSREYYYLGTQLPIDRFLTFYYG GRIKHTEYYQCGKGRDLGFGTILWFQTKIGTGMGEQMLSREYYYLGTQLPIDRFLTFYYG GRIKHTEYYQCGKGRDLGFGTILRFQTKIGTGMGEQMLSREYYYLGTQLPIDRFLTFYYG GRIKHTEYYQCGKGRDLGFGTILWFQTKIGT GMGEQMISREYYYLGTQLPIDRFLTFYYG GRIKHTEYYQCGKGRDLGFGTILRFQTKIGTGMGEQMLSREYYYLGTQLPMDRFLTFYYG ARIKHTEYFQCGKGRDLGFGTILFFQTKIGT GMGEQMLSREYYYLGTQLPIDRFLTFYYG

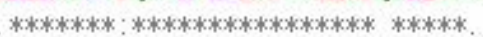

-PDMLVILSVQIFIVTMVFLGTLNDLLVCKYSSSGQFIG-TTGCYNLTPAFQWI HPGFH MRMLVILSVQIFILTMVFLGLLGQLQVCKYSPSGQLIS-PTGCYRLTPAFQWI

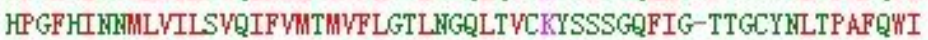
HPGFH KIIMLVILSVQIFILTMVFLGTLAGQLICKYSSSGQLLG-PTGCYKLTPAFQWI HPGFHШMIMLVILSVQIFIVTMVFLGTLFGLKLCQYSKSGQLLG-PTGCYHLTPAFQWI HFGFH MIMLVILSVQIFILTMVFLGTLNGQLEICKYTSSGQLIG-PTGCYKLTPAFQWI

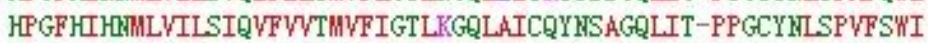
HFGFH INIMLVILSVQLFIVTMVFLGTLASELTICQYTSSGGLIGGQGGCYKLHFVFDWI HPGFHШMIMLVILSVQLFIVTMVFLGTLISSVTICRYTGDGQFIGGQAGCYHLDPVFDWI

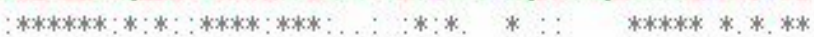

DHCIISIFLVFMIAYLPLFLRELVERGTGKAVFRLAKGFGSASPAFEVFSTQISSHSIIT DHCIISIFLVFMIAYLPLFLQELVERGTVKAVVRLAKYFGSLSPAFEVFSTQISSHSIIT DHCIISIFLVFMIAYLPLFLQELVERGTVKAVIRLAKFFGSLSPAFEVFSTQISSHSIIT DHCIISIFLVFMIAYLPLFLLELVERGTVKALFRLAKGFGSLSPAFEVFSTQISSQSIIT DHCIISIFLVFMIAYLPLFLQELVERGTIKAVFRLAKHFGSLSP AFEVFSTQIYSHSIIT DHCIISIFLVFMIAYLFLFLLELVERGTIKAVIRLAKQFGSLSPAFEVFSTQISSHSIIT DRCIISIFLVFLIAYLPLFLQELVERGTGKAILRLTKFF ASLSPAFEVFSTRISSHSIAS DRCIIGILLVFMINFLPLFLQELVERGTGRAIFRLGKQFLSLAPVFEVFSTQIYTHSILS KRCILSIFLVFMIAFLFLFLQELVERGTWKAIFRLAKQFGSLSPVFEIFSTQIYTHSILS

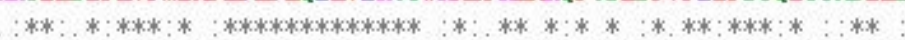

HLTFGGARYIATGRGFATTRISFSILYSRFAGPSIYLGMRTLIMLLYVTLTIWTGWVTYF RLTFGGARYIATGRGFATTRISFAILYSRFAGPSIYLGMRTLIMLLYVTLTIWTGWITYF RLTFGGARYIATGRGFATTRISFAILYSRFAGPSIYLGMRTLVMLLYVTLTIWTGWTTYF HLTFGGARIATGRGFATTRISFAILYSRF AGPSIYLGMRTLVMLLYVTLTIHTGWVTYF BMTFGGARYIATGRGFATTRISFAILYSRF AGPSIYLGMRTLVMLLYVTLTIWTGWVTYF HLTFGGARYIAT GRGFATTRISF AILYSRF AGPSIYLGMRTLIELLYVTLTIWTGWVYYF RLTFGGARYIATGRGF ATSRISF AILYSRF AGPSIYIGMRTLVMLLYVTLTLW'TG"WTYF RLAFGGARYIATGRGFATSRISFSILISRF AGPSIYLGMRTLUMLIYVTMSLWTPFLIYF WLTFGGARYIATGRGF ATSRIYFNILFSRF AGPSIYLGMRTLVMLLYVTLSLWTPYLIYF

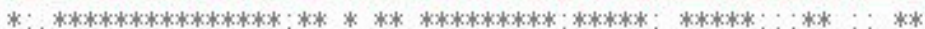

1108

1126

1124

1123

1121

1124

1115

1116

1118

1168

1186

1184

1183

1181

1184

1175

1176

1178

1200

1246

1244

1243

1241

1244

1235

1236

1238

1253

1305

1303

1302

1300

1303

1294

1296

1298

1313

1365

1363

1362

1360

1363

1354

1356

1358

1373

1425

1423

1422

1420

1423

1414

1416

1418 
Gf 1,3-beta-glucan svuthases $\mathrm{Pb}$ 1, 3-beta-glucan syuthase Tv 1,3-beta-glucan sunthases Gl 1,3-beta-gugucan șuthases Ds 1,3-beta-glucan syuthases Lt 1,3-beta-glucan synthases Or 1,3-beta-glucan synthases Gt 1,3-beta-glucan synthases Ag 1,3-beta glucan synthases

Gf 1,3-beta-glucan svuthases $\mathrm{Pb}$ 1,3-beta-glucan syuthases Tv 1,3-beta-glucan swuthases G1 1,3-beta-givocan șuthases Ds 1,3-beta-glucan syuthases Lt 1,3-beta-glucan synthases Or 1,3-beta-glucan synthases Gt 1,3-beta-glucan synthases Ag 1,3-beta glucan synthases

Gf 1,3-beta-glucan swnthases $\mathrm{Pb} 1,3$-beta-glucan syuthases Tv 1,3-beta-glucan snuthases G1 1,3-beta-glucan syuthases Ds 1,3-beta-glucan synthases Lt 1,3-beta-glucan synthases Or 1,3-beta-glucan synthases Gt 1,3-beta-glucan syuthases Ag 1,3-beta glucan synthases

Gf 1,3-beta-glucan svnthases $\mathrm{Pb} 1,3$-beta-glucan synthases Tv 1,3-beta-glucan swithases G1 1,3-beta-glucan synthases Ds 1,3-beta glucan synthases Lt 1,3-beta-glucan syuthases Or 1,3-beta-glucan syuthases Gt 1,3-beta-glucan syuthases Ag 1,3-beta glucan synthases

Gf 1,3-beta-glucan swuthases $\mathrm{Pb}$ 1, 3-beta-glucan synthase Tv 1,3-beta-glucan sunthases G1 1,3-beta-glucan syuthases Ds 1,3-beta-glucan synthases Lt 1,3-beta-glucan synthases Or 1,3-beta-glucan synthases Gt 1,3-beta-glucan syuthases Ag 1,3-beta-glucan synthases

Gf 1,3-beta-glucan svinthases $\mathrm{Pb}$ 1,3-beta-glucan syuthases Tv 1,3-beta-glucan swithases Gl 1,3-beta-glucan syuthases Ds 1,3-beta glucan syuthases Lt 1,3-beta-glucau synthases Or 1,3-beta-glucan synthases Gt 1,3-beta-glucan synthases Ag 1,3-beta glucan syuthases
"WVILALCISPFLFRP HQFSF ADFIIDYRVLAMDEPRQLAR--TPEFLD"'WL---LPVITD "WVILAMCVSPFLFHPHQFSAАDFIIDYREFVRWMHRGKSRАHАHSWIGYCRLSRTMITG "WSILALCVSPFLFNPHQFSARDFIIDYREFLRWMHRGISR丸HんHSWIGYCRLSRTMITG "VSILALCVSPFLFNPHQFSAんDIIDYREFLRWMHRGKSRAHんHSWIGFCRLSRTMITG "VSILALCVSPFLFNPHQFSPADFIIDYREFLRWMHRGNSRAHAHSWIGYCRLSRTMITG "YSILAMCVSPFLFHP HQFSAADFIIDYREFVRWMHRGHSRAHАHSWIGYCRISRTMITG "WYSVLSLCVAPFLFWPHQFSF ADFVIDYREFLRWMHRGKSRYHVISWIGYCRLSRTMITG "ISTLALCIAPFLFNP HQF ALSDFVIDYREFLRWMCRGKSRSHRHSWIGYCRLSRTMITG "ISIVALCVAPFLFRPHQFYFSDFIIDYREFLRWMSRGKSRSHRHSWIGYCRLSRTMITG

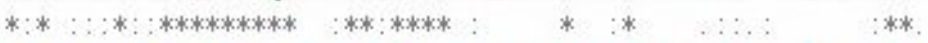

YDYRLQEEEARASSEKISGDVPRAGWRAVLFEVIFPIVMATLFVIAYMFVKSFPDKDGK Y----KKKRLGHPSERLSGDVPRAKHRAVIFSEVVFPVMATLFVIAYMFVKАFPDKDGK Y----KKKRLGHPSERLSGDVPRAKWRAVIFSEVIFPLVMAVLFTIAYMFVKSFPDKDGK Y----KKKRLGHPSERLSGDVPRAKWRAVIFSEVTFPIVMATLFVIAYMFVKSFPDRDGK Y----KKKRLGHPSERLSGDVPRAKWRAVISEVIFPIVMAILFVIAYMFVKSFPDKMGK Y----KKTRLGHPSERLSGDVPRAKWRАVIF АEIIFPIVMАVLFVI АYMFVK丸FPDKDGK Y----KKKRLGHPSEKISGDVPRAGWRAVIFSEVMFPIVSATLFVVCYMFVKSFPDKMGE Y----KKKKL GLPSEKISGDVPRAGWRAVIISEIVFPIVMAVLFVIAYMFVKSFPDRHG Y----KKKKL GHPSEKISGDVPRASWHAVIFSEVVFPVIMAILFTIAYTFVKSFPV-DGE

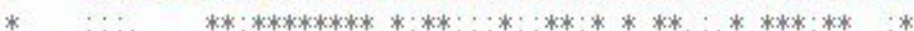

QPPSPLIRIAIVSLGPIVWRАILLVLFMFSLFLGPMLDTPFPKFGSLMAFLGHSLGVIG QPPSPLIRIA工VSLGPVN"AнILLVLFMFSLFLGPMLDTPFPKFGSVMAFLGHSLGVVG QPAגPLIRIAVVAIGPIVHAАAILLVLFMFSLFLGPMLDTPFPKFGSVMAFLGHTLGVVG QPPSPLIRIAIVSIGPIVW RАVLLVFLFSLFLGPMLDTPFPKFGSVM QPPSPLIRIAIVSIGPIVHKAИLLVLFFSLFLGPMLDTPFPKFGSLMAFLGHTLGVIG QPPSPLIRIAIVSLGPVWWARVLLVFLFSLFLGPMLDTPFPKFGSVMAFIGHSLGVVG QPPSPLVRIAIFSVGPMVHKAHLLVQFMVSLFLGPLIDTPFPKFGSVMAFLAHTLGVVG QPPSPLIRTAVVSGPIVWAGVLLVLLISLFMGPMLDSVFPKFGSVMAFIAHFLGTVG TPPSPLVRIAVISLGPIVHНАILLTLFLISLFLGPMLDPLFPLFGSVMАFTАНLGАVG

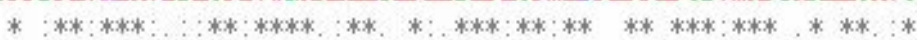

MIAFFEFL"FLEL"WVAHAVLGIAVIFIQRAIHKVLISVFLSREFKMETKRA"N"TGKW

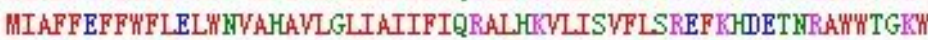

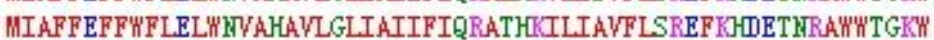

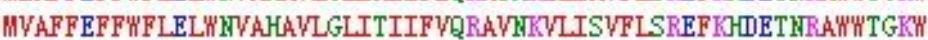
MIAFFEFF"'FLEL"WVAHAVLGLTIIFIQRAVKKVISVFLSREFKWETHRA"W"WGKW" VIAFFEFF"FLELWWVALALGLIAIIFIQRALHKVLISVFLSREFKOETKRAW"WTGKW MIGFFEFF"YLEL"WWSHAVLGLVIISIQRAIKKVFIAVFLSREFKTETHRAW"WGKW

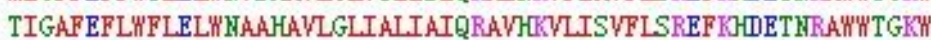
MVGFFEFL" "FLEL"WVSHAVGLITVISIQRAVIKILISVFLSREFKMETKRA"WTGR"

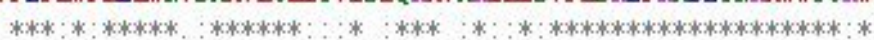

YGRGLGSHAMSQPAREFIVKIIELSL"WSSDFLTGHLLFLLTPPILIPYFDRLHSTMLF" YGRGLGAHAMSQP AREFIVKIIELSLWSSDFLIGH.LIFILTPPILIPYIDRLHSMLIFW YGRGLGSSAMSQP AREFIVKIIELSLWSSDFLIGHLLFILTPPILIPYIDRFHSMMLFW" YGRGLGAHAMSQP AREFVVKIIELSL"WSSDFLMGHLMFILTPPIVIPYIDRLHSMMLFW YGRGLGSHAMSQPAREFVVIIELSL"WSSDFLMGHLMFILTPPVLIPYMDRFHSMMLF" YGRGLGAHAMSQPAREFIVIIELSLWSSDFLIGHLLFLLTPPILIPYIDRLHSMMLFW" YGRGLGSHVMSQPAREFIVKIIELSLWSSDFITGHFLLFILTPPILIPYIDRAHSTMLFW YGRGLGHLAMSQP AREFIVKIVELSL"WSDLLIGHFLI.FMLAP AALMPFMDKIHATMLFW" YGRGLGAHVMSQPAREFIVKIIELSLWSSDLIIGHLIFMLTPPILVPYFDRFHATILFW

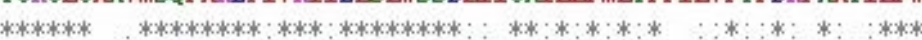

LRPSKQIRAPLYSIKQKRQRRWIVIKYGAVYVLAIGIFVALTVIPLVFRQSLTFHCSICQ QV LRPSKQIRAPLYSIKQKRQRRWIIIKYGFVYVAFATFIALIAVPVIFRDULTFWCSICQ GI LRPSKQIRAPLYSIKQKRQRRWTVIKYGFVFVLAVALFАAMLVIPLIFRQSLHVRCTICQ SI LRPSKQIRAPLYSIKQKRQRRWIVVKYVVILGF AIFGALIAIPLVFRQHLTLWCSICQ GI LRPSKQIRAPLYSIKQKRQRRWIVIKYGVIYVLGF AVFGALLALPLAFRDHLTISCSICQ GI LRPSKRIRAPLYSIKQKRQRRWIIVKYGILYILAF AIFVALIAVPLVFRQDLTISCSICQ SI LHPSKQIRAPLYSMKQKRQRRWIVIKYGIVYLIGIGIF AVLIALPLVFRHSLRL.KCSICQ TI LRPSKQIRAPLYSIKQKRQRRWIVIKYGTVFVAАLTLFIALIALPAнFRDRILFHCSVCN SL LRPSKQIRAPLYSIKQKRQRRWIIIKYGTIYIAVIALFVLIIALP AVFRTTLFFDCSVCH SL

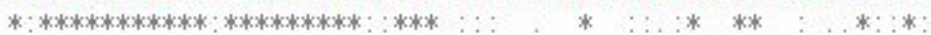


Figure S3 Amino acid sequence alignment of $\beta$-1, 3-glucan synthases from fungi including Grifola frondosa (accession number: MK808019 Gf), Polyporus brumalis (accession number: RDX52365.1, Pb), Trametes versicolor (accession number: XP_008043357.1, Tv), Ganoderma lucidum (accession number: AZQ26797.1, Gl), Dichomitus squalens (accession number: XP_007367299.1, Ds), Lentinus tigrinus (accession number: RPD60614.1, Lt), Obba rivulosa (accession number: OCH86741.1, Or), Gloeophyllum trabeum (accession number: XP_007869676.1, Gt) and Armillaria gallica (accession number: PBL03082.1, Ag). 


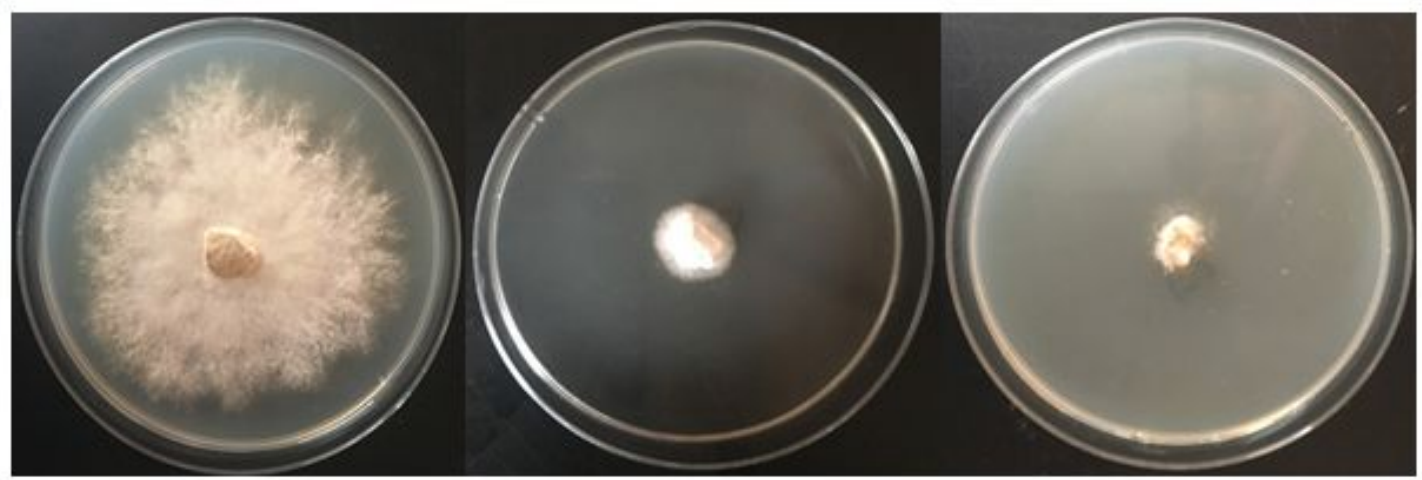

$$
0 \mu \mathrm{g} / \mathrm{mL} \quad 50 \mu \mathrm{g} / \mathrm{mL} \quad 60 \mu \mathrm{g} / \mathrm{mL}
$$

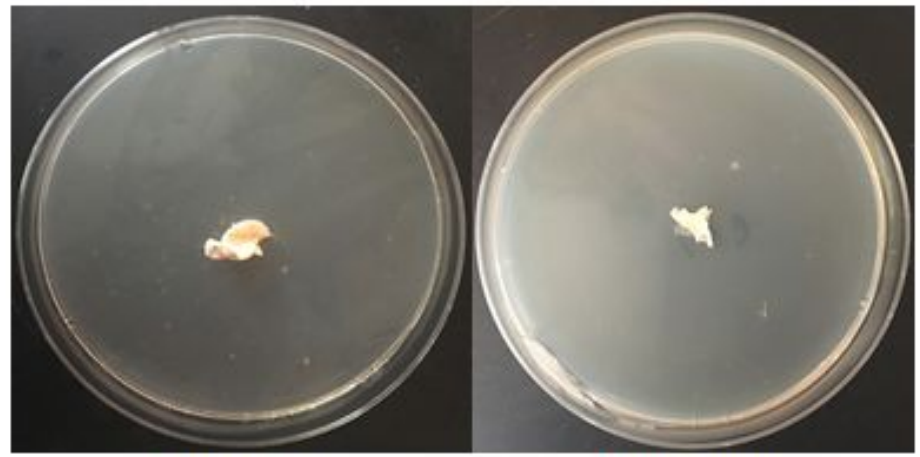

$80 \mu \mathrm{g} / \mathrm{mL}$

$100 \mu \mathrm{g} / \mathrm{mL}$

Figure S4 Effect of different concentration of hydromycin B on the mycelial growth of $G$. frondosa. 
>Analysis of functional domain prediction of Dicer-like protein 1 in G. frondosa

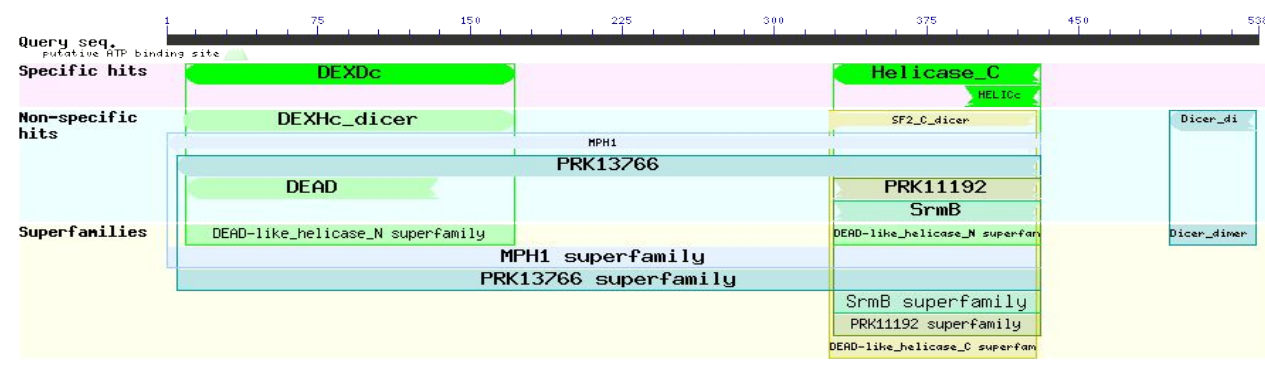

> Nucleotide sequence of Dicer-like protein 1 [2027 bp, LUGG01000004.1:1754000-1756026 ] ATGACTGTGGATATGGACGTCCTTATACCTCGCCGGTATCAAGAGGAAATATTCGTAC GTGCTCAACAGGGCAACGTCATCGCCGCACTGGACACCGGAAGCGGCAAGACATACA TTAGCACGCTCCTCATCAAATGGGTAGCCGCAAGAGACATAGGGCTGGGGAGGATTA TCGTATTTCTCGTTCCAAAAGTCGCCCTCGTACAACAGCAGGGCGATTTTATCGCTCA ACAGACTCCCCTCCGCGTCAGCCAGTTTTGTGGCGCTACCGCGATGGATATGAGCGAT CGCGCAGGTTGGAAGAAACAACTCTCAGGCTCAGACGTGTTTGTCATGACAGGTATA CTATCCAGCTGTCATTGTGATTGGTCATCTACTCATCACCGATTCTCAGCTCAGATATT TCTCAATATCCTCACTCATTCGCATTGGAGTTTAGAAAATGTACACGTCATTCTTTGTT CCAGATACGCCATCTGAATTATTTTTGTTCCAGATTTCTCTCATGGTCTTCGACGAATG TCATCATACCCGGAAGAATCACGCATACAACGGTATAATGCGCGAATATTTTAACTTC CTTCAGGAAAGCGGCCCAAGGTGTTCGGTATGACCGCGTCCCCGATCTGGAACCCGA AGGACGCCACGGAATCACTCGCCACCTTGGAGCGAAATTTGGATGCCAAGGTCATCG CCGTTCGAGACCATGTAGACGAGCTCATGGGACATTCTCCGAAGCCTGAAGAGGTGA TCCACGAATATCCGCTGCCTCCAGACTTGTATCCCGAATACCCCGGCCACTCCCTCTG GCAGCGTCTGAGCTTAGGCTTTTTACCCGCGGAGATCGACATACCCGTAGAAAGGAT TATGACACGATACCAGGTCGCGAACCACAGCTTAGGGCCATACGCTGCAGACCTCTA TCTCTATCTGGACATCAAGCAACGTGTCGAGCAGCTCGTTCACAATGCGCATGAGGC GGACATGTCATTTCTTGCGCTCACACAGTTTGTCACCAAGGACGGCGACCACGGTTCG AATATAGAGAATAGCACCCTGCATCCGCGAGTTAAGCAGCTCCAGAATGTGCTGAAG GAATGCAGCCATCTATTCGAGGATGAGTCAAACCCAGATATGGTACCGGTCGAGGTG CCCCTCAAATGGTGCTCGCCGAAATTGCGGATGCTGGCTGACATCCTGTTCGCTCATT ACACGGCGACATTCCAAGGAATTGTGTTTGTCGAGCAGCGTCATGTTGCGGCGTGCCT CGCGAGGATCCTCCCTTGCATTCCGCAGTTGAATCACCTTATCAGGTGTGGGCAGCTT ATAGGCCATGGCGCTTCTCACACAGCGAGTGCCCACCTCAAGGGCATGGCTCTCAAG ACGCAGCAGGATAGTGTGAACTTTTTTCGAAAGCGCCAAGTGAACCTTCGTAAGTAA TCCGCAACTCTCATTTGCCACATTTGCTGATTATTTCGTTTGCTGCAGTCATCGCAACC TCTGTGGCCGAAGAAGGCTTGGATTTTCCCGTGAGTGGTTTCTGTTTGCTTGTCACGA GCAGGATTCATTGTTCGTCAGGCTTGCGATCTCGTTATACGGTTTGACCCCATGCAAC ACATGGTCGGCTACCTACAATCGCGGGGGGGGCACGCCACAAGACATCGACCTTCGT CATCATGCTCCCGCAAGGTCAGAGCGTTGACAAGGACCGCTACAGGGCGTTCTCGGA AAGCGAGCCCCAACTCCGCATGGTTTACCAGATGCGTGAGGACGCCGCGAAGAAGGC CGAAGAGGCGGAGGAGGCGGAGGACCACGATGATCCCGCCGATATCCTCGAGAGGG AGCGATTCGTTGTGCCCTCAACAGGTGCCGTCCTTACATACAATAGCTCCATCAGCCT 
TCTCGGTCATCTGTGCTCTTTAATACCCCGGGACCGATTCACCCCCGTCCAGCTACCG AAATATTCTGGAAACAATGTTGCTACTGTACGCCTTCCATCCAGTCTCCCTTACCTCCC GATCTTCTAG

$>$ Amino acids sequence of Dicer-like protein 1 [538aa, OBZ75102.1]

MTVDMDVLIPRRYQEEIFVRAQQGNVIAALDTGSGKTYISTLLIKWVAARDIGLGRIIVFL VPKVALVQQQGDFIAQQTPLRVSQFCGATAMDMSDRAGWKKQLSGSDVFVMTAQIFLN ILTHSHWSLENISLMRPKVFGMTASPIWNPKDATESLATLERNLDAKVIAVRDHVDELMG HSPKPEEVIHEYPLPPDLYPEYPGHSLWQRLSLGFLPAEIDIPVERIMTRYQVANHSLGPYA ADLYLYLDIKQRVEQLVHNAHEADMSFLALTQFVTKDGDHGSNIENSTLHPRVKQLQNV LKECSHLFEDESNPDMVPVEVPLKWCSPKLRMLADILFAHYTATFQGIVFVEQRHVAACL ARILPCIPQLNHLIRCGQLIGHGASHTASAHLKGMALKTQQDSVNFFRKRQVNLLIATSVA EEGLDFPSVDKDRYRAFSESEPQLRMVYQMREDAAKKAEEAEEAEDHDDPADILERERF VVPSTGAVLTYNSSISLLGHLCSLIPRDRFTPVQLPKYSGNNVATVRLPSSLPYLPIF

Figure S5 Analysis of functional domain prediction, Nucleotide sequence and amino acid sequence of Dicer-like protein1 in G. frondosa 
>Analysis of functional domain prediction of Dicer-like protein 2 in G. frondosa.

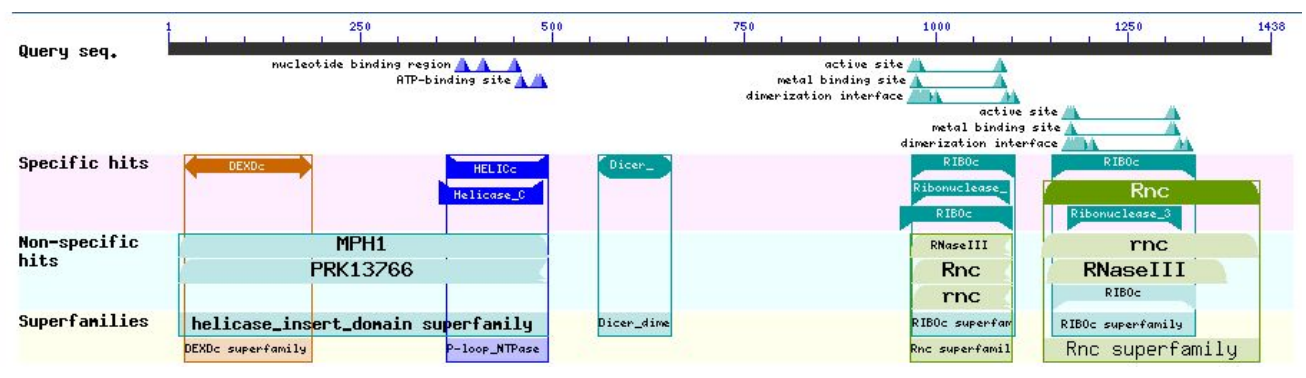

> Nucleotide sequence of Dicer-like protein 2 [4773 bp, LUGG01000019.1:407719-412492 ] ATGTCTGTTTCAGAAGCAGATATAGAAGCAGAAGCTTCAGCCCCACCAGTTCTGCAC ACCAGAGCGTACCAACAAGAGCTGCTGGAAGAGAGTCTCCGCCGAAACCTCGTCATC GCGCTGGACACTGGTTCTGGTAAGACGCACATCGCCGTCCTCAGGATGAAGCTAGAA GCTGAGCGCAATTCGAACAAGGTATGAGGCGCATATAGTCACCATGCGTCAACTCAT GAAGAGGACATGACAGATCTCGTGGTTTATTGCCCCCACTGTTGTCTCGTTGAGCAAC AGCGCGAGGTGATCGCCACGGCCGTACCGGTTTCCGTCGGTCTGATCTCGGGCGCGTC AGAACACAGGCCAGTGGAAGAATGTCGCATTGTGGCGGTCCATTCTCGAGACTCATC GTATCATGGTGACGACGCCACAGATTCTGCTGGATGCCTTGCACCATGTGAGTTCCAC TGGCCATCCTTATTTCCCTTCTTCGGTGCGCTCATTCCTTACATAGGGCTACATTGACC TTGGACGGGACATTGGGCTATTGGTGTTCGATGAAGCTCACCATGCAATTGACAAAC ATCCCTACAATATGATCATGAAAAATTTCTATTTCAACCTCCCGTTGCGCACAGGCCA AGAAGACCTACAAGCGCGCGTGCGACCAATGATCCTCGGTCTTACAGCAAGCCCTAT ATATGGTGGTAATGTCGACAATTCCTTCTAGTAGGTCATCGCACGGGATTTCCAAATA TCCAAGGTTAACGATGATTTATAGCAAACTAGAGAAGAACCTTGACAGCATCATTCG CTCGTCGCGCGTGAACCGGAATGAATTGGCGCAGTATGTTCATCGCCCAGTATTCAAG CATGTGCTGTATCCGATGTCAAACCAAGATTGGGAAACTATGCCGTCAGAGAACCAT CGTGCGTTGCAGGCGATGGTCGACGGAATGAATATCGAGGATGATCCGTACGTGAAG TCGCTACGCGAGCGGCTCGCCAAACTTCCTCCTGGTGACGAACGCACCCGTGTCGACC AGCAGCTGTCAAAGACAATTAACAAACAGGACACGTTCACCCACAAGGGCCTTCGGG ACTTCGCTCGAACTGCGGGGGATATTTGTTTTGAACTCGGCACATGGGCTGCGGATTG GTATGTTGAACAGGTCATTAGGCAGGCGAGGATCGCTGCAAATCCGTACAACAATAT CATGTCTGCCTGGCAGGAAAAGGAGAAGAGGTATATATTGGATGCACTCTCGAGCGT TCAACTCGTGCCTGTGTCCTCCGACCCTCGTGACATTCTTGCCGGCATCTCCCCGAAG GTCCGTGCGTTGGTTGACTGTCTTACCCTGGAGGAGGTGCTTTTCCGGTCGCAGGACG AAGATTACAGCGGCCTTGTCTTCGTGACGCGCCGCGACACAGTGCTCGCGCTTGCTGA GGTTCTTTCTCGGATCCCAGAGACCGCGCAGCTCTTCCGGATTGGCTACCTTCTCGGA AGCTCCTCGAGTTTCAAGCGGCACGCATTTCTGGATATCACGCGCATGATGCTGAAAG ACTCGCAGTCGGACACGCTGCGCGACTTTAAGATCGGGGATAAGAACCTGATCATCT CGACCTCGGTAGCGGAAGAAGGGATAGATATCCAGGCGTGCGGGAGCGTGATACGCT TCGATCCGCCACCTAACGTCGTCGCTTGGGCGCAGAGCCGGGGGCGGGCGCGGCGGA AGCGGAGCAGTTTTGTGATCATGTTCGAGGAGTCAGGTGTGCACCAGAAGCTCGTTG 
AGCAGTGGGAAAATGCGGAGAAACAGATGATGGCGTTATATAATGATATGCAGAGG GATTTGAAGGGTGCAGGGAACGAGAACAATGATGAATTCGATGGAGGGGATGGTTAT GTTGAGTTTGAAGTCGCATCAACGGGGTGAGTCTTACGCGATATGAAAGATTTCCTGG TGACTGACTTCTCTCTCATAAGTGCTCTTCTCACTTTGCATTCTGCCACTAGTCATCTA AATCATTTTTGCGCCGTCATCCCGAACGCCGGTCATGGCGGTCATGTCCCCATCTATG ACCTTGACCCTCCTGATTATAGAGAGAATTGGCATTCCTCCCCAGATCAATCACTCCA GCGCTATGGGGGCCCGTGGGGAGCGACTGTCTCGCTCCCCAGGGTCCTTCCTCCAAG GTGCGCATTTCGTTAGGAACAATGATGATGATATTGCTGACCACAAATGTACTTGCTG CCAACCAGCTTGCGTAAGTTTTCGACCGAATGTATTCACAGGACGAAGCGAGCGGCC CAGCAGCATGCGGCCTTCAAAGCTTATGTTGAGCTGTACAAGGCCAAACTCCTAAATT CACATCTCCTTCCTTTGACAAGCGTCGTTGAACCTGATAAAGAGGAAGAAGTCAAGA GGCTTCTCCAGGAGATCGAGAAGCGAGCAGGTACCGCCAAAGTGACCATACAAATGG ACCCTTGGGCACCTAAAACGGACTCGCCGGCGTGGTTCGTAAACGAACTAGAACTAC AAGGACTCCCCGCGTTGCGTATGCTCACTCGCAATCCGCTATCCACGTTTTTAGAGCA AGATCTTCCATGCCTTTATGTTCCTGGTAGGGCGCCGATCAAAGTTCGCATTCGACAT CAGAGTACACTTGATGCAGGAGATGCATACATCGAGCGTGCTCGCCGTTATACCTAC GATCTGTTTACAAGACTCTATGGTGCTCGTATGGATCCCGAGAATATCTCATTCTCCT ATTTGTTCCTTCCCGTGGTCGAGGGTATCCACGAACAGAAGTGGATCGAGCGCCGAA CTTGGCAACGGGATCGCTGTGATCGCTCTGTAACAGTGCGGTTAGAGAATTCTGCAA GGGCTAATGCAGAGATCCTTGGCGAGCAGTTCTCTCACCCCTCTGACTTAGCTCTAAT CAGGAATAATGCCAAATTTGACAAACCTCTTCGATTCGTCCAATGGCACTACGAAGC CATCACGTCAGAGCAGGAGGAGGAGCTACGAGAGATATACGAGGAAGTTCCGGATA TGCGTATCTCGTATCCCTTGCTCCTGGTGAAGCCTTTCCCTCGGCGAAGGAACTTCCTT GTGCCCCTCTCTTCTGAGACCGAAGGGCTTGGACACGAGGAACCGTTTCTTCTTCATC CCAAATACTCGACTATCGATCTGGTGTCAAGCGATGATATTGAATTCGCCATGCTCCT CCCGTCCTTCCTTCGGTGGTTGACCAATGCGCTCACAGTCGCTTCCCTCCGCGAGAAT CTTCTCGTTGGTACCCCCGTATTCCGCATCCCCTGCCTCTTCTTACCACAGCGGTCACT GCACCCGTCTCTCAGGAAGCTCTGAACTATCAGCGGCTGGAAACTTTGGGAGACACT GTCCTGAAGTTCATTGTCAGCTTGCAGCTCTTTGCAGACAACCCGCTCTGGCATGAGG GCTATCTGAGCAGAAGAAAGGATCACGTCGTGGCCAATTCTAGTCTGGCAAAGGAAG CTATCGACAAACGTCTCTATCAGTGGATTCTTCGCGATCGTTTTGTACCAAGGAAGTG GAAACCTCACTACCATTCGGAAGCTTCCAGCGATGACTCCAAGGAAGACACAGAACC CACAGAGGCGGTCTCTGATTCCCGTGACAAGAAGAAAAATAAGAAGGCAGAGGATC TCTCCACGAAAATGCTGGCGGATGTCGTAGAGTCATTGATCGGTGCAGCGTACGAGC ACGGCGGATTTGATCTCGCCATTGACTGCGCGCAGATATTCGGACTCGGTATCTCTTG GCAGAAGCTTCCGCTTCGTATGGAACGCATCTTAGCAAACACAGAAAACCTGGACGA TCTTCCTCCTGAACTCAACCTTGTCGAATCCATGCTCGGATATCAATTCTCTCGACGA ACACTCCTTGTCCAGGCTTTGACTCACTCCAGCTACCATGGCGATATTGCTTCTATTTC TCTAGAGAGATTGGAGTTCCTTGGCGATTCCGCCCTAGACATGGTTGTAACAGATTTT CTGTATCATGCACCCGGTAAGAATTATACCCCAGGACATATGCACATGCGCAAAGAG GCCACGGTCAACTCACACTTCCTTGCATACATCTGTCTGAGCACATCGACGGTCGTAG ACGGTACCATGCCATTCTGGACCAAAGATCACGGTCTCACGGTCTCTACTGAATCACA TCGCATTCACCTTTGGCAGTGTCTCCTGCATTCGAGCCATCGCGTTCTAGATGACCAG AATGTCGCGTTTTTGCGTTACCAGAAAAGTGGTCCCACCATCACTCATGCCATGCAGA 
ATGACGCCATATACCCATGGGCCGCCTTAACCAGCCTGCAGGCCCCCAAATTTATCAG CGACATGGTCGAAAGTCTTCTAGGTGCTGTCTACGTGGATTCAGTCGGGAATCTAGAC GTCGTGCGTGCTGTGATGAGGACCCTCGGAATCATGTCGATCATGGAGCGGATTGTG GAATCAGAGGTTGACGTCCTGCATCCCGTCTCCCGCCTCGCGATGTGGGCAGATCAAC AGGATCCGAAACAGAAGGTAGAGTATGTGTTCAAGAAGGAGGATGGTAATGTTAGCT GCGCGATCATGCTAGATGACGTGGAGGTCGTCAAGGTCACAGAACCGTACCGCAGCC GGGCGAGCCAGGAGGAGGTGAGGTTTGCGTCTGCTGAAAAGGCAATCAAACTCTTGC ATGTCTGTGACGAGGAAGAGGTCGAGGGTTGGGGTGATATACCGGAGTATGACTGA

>Amino acids sequence of Dicer-like protein 2 [1438aa, OBZ68881.1]

MSVSEADIEAEASAPPVLHTRAYQQELLEESLRRNLVIALDTGSGKTHIAVLRMKLEAER NSNKNTGQWKNVALWRSILETHRIMVTTPQILLDALHHGYIDLGRDIGLLVFDEAHHAID KHPYNMIMKNFYFNLPLRTGQEDLQARVRPMILGLTASPIYGGNVDNSFYKLEKNLDSIIR SSRVNRNELAQYVHRPVFKHVLYPMSNQDWETMPSENHRALQAMVDGMNIEDDPYVK SLRERLAKLPPGDERTRVDQQLSKTINKQDTFTHKGLRDFARTAGDICFELGTWAADWY VEQVIRQARIAANPYNNIMSAWQEKEKRYILDALSSVQLVPVSSDPRDILAGISPKVRALV DCLTLEEVLFRSQDEDYSGLVFVTRRDTVLALAEVLSRIPETAQLFRIGYLLGSSSSFKRHA FLDITRMMLKDSQSDTLRDFKIGDKNLIISTSVAEEGIDIQACGSVIRFDPPPNVVAWAQSR GRARRKRSSFVIMFEESGVHQKLVEQWENAEKQMMALYNDMQRDLKGAGNENNDEFD GGDGYVEFEVASTGALLTLHSATSHLNHFCAVIPNAGHGGHVPIYDLDPPDYRENWHSSP DQSLQRYGGPWGATVSLPRVLPPRTKRAAQQHAAFKAYVELYKAKLLNSHLLPLTSVVE PDKEEEVKRLLQEIEKRAGTAKVTIQMDPWAPKTDSPAWFVNELELQGLPALRMLTRNP LSTFLEQDLPCLYVPGRAPIKVRIRHQSTLDAGDAYIERARRYTYDLFTRLYGARMDPENI SFSYLFLPVVEGIHEQKWIERRTWQRDRCDRSVTVRLENSARANAEILGEQFSHPSDLALI RNNAKFDKPLRFVQWHYEAITSEQEEELREIYEEVPDMRISYPLLLVKPFPRRRNFLVPLSS ETEGLGHEEPFLLHPKYSTIDLVSSDDIEFAMLLPSFLRWLTNALTVASLRENLLVAVTAP VSQEALNYQRLETLGDTVLKFIVSLQLFADNPLWHEGYLSRRKDHVVANSSLAKEAIDK RLYQWILRDRFVPRKWKPHYHSEASSDDSKEDTEPTEAVSDSRDKKKNKKAEDLSTKML ADVVESLIGAAYEHGGFDLAIDCAQIFGLGISWQKLPLRMERILANTENLDDLPPELNLVE SMLGYQFSRRTLLVQALTHSSYHGDIASISLERLEFLGDSALDMVVTDFLYHAPGKNYTP GHMHMRKEATVNSHFLAYICLSTSTVVDGTMPFWTKDHGLTVSTESHRIHLWQCLLHSS HRVLDDQNVAFLRYQKSGPTITHAMQNDAIYPWAALTSLQAPKFISDMVESLLGAVYVD SVGNLDVVRAVMRTLGIMSIMERIVESEVDVLHPVSRLAMWADQQDPKQKVEYVFKKE DGNVSCAIMLDDVEVVKVTEPYRSRASQEEVRFASAEKAIKLLHVCDEEEVEGWGDIPE YD

Figure S6 Analysis of functional domain prediction, Nucleotide sequence and amino acid sequence of Dicer-like protein 2 in G. frondosa 